\title{
Normality and Gap Phenomena in Optimal Unbounded Control ${ }^{\text {th }}$
}

\author{
Monica Motta \\ Dipartimento di Matematica, Università di Padova \\ Via Trieste, 63, Padova 35121, Italy \\ Telefax (39)(49) 827 1499, Telephone (39)(49) 8271368 \\ Franco Rampazzo \\ Dipartimento di Matematica, Università di Padova \\ Via Trieste, 63, Padova 35121, Italy \\ Telefax (39)(49) 827 1499, Telephone (39)(49) 8271342 \\ Richard Vinter \\ Department of Electrical and Electronic Engineering, \\ Imperial College London, Exhibition Road, London SW7 2BT, UK \\ Telefax (+44) 2075946282, Telephone (+44) 2075946287
}

\begin{abstract}
Optimal unbounded control problems with linear growth w.r.t. the control, both in the dynamics and in the cost, may fail to have minimizers in the class of absolutely continuous state trajectories. For this reason, extended versions of such problems have been investigated, in which the domain is extended to include possibly discontinuous state trajectories of bounded variation, and for which existence of minimizers is guaranteed. It is of interest to know whether the passage from the original optimal control problem to its extension introduces an infimum gap. This will reveal whether it is possible to approximate extended minimizers by absolutely continuous state trajectories, as might be required for engineering implementation, and whether numerical schemes might be ill-conditioned. This paper provides sufficient conditions under which there is no infimum gap, expressed in terms of normality of extremals. The link we establish between infimum gaps and normality gives insights into the infimum gap phenomenon. But, perhaps more importantly, it opens up a new approach to devising useful tests for the absence of infimum gaps, namely to supply verifiable sufficient conditions for normality of extremals. We give several examples of the use of this approach, and show that it leads to either new conditions, or improvement of known conditions, for no infimum gaps. We also give a criterion for non infimum gaps, which covers some problems where the normality condition is violated, illustrating that sufficient conditions of normality type, while covering many cases, are not necessary.
\end{abstract}

\footnotetext{
"This research is partially supported by the INdAM-GNAMPA Project 2017 "Optimal impulsive control: higher order necessary conditions and gap phenomena"; and by the Padua University grant PRAT 2015 "Control of dynamics with reactive constraints"

${ }^{*}$ M. Motta

Email addresses: motta@math.unipd.it (Monica Motta), rampazzo@math.unipd.it (Franco Rampazzo), r.vinter@imperial.ac.uk (Richard Vinter)
} 
Keywords: Optimal Control, Maximum Principle, Impulsive Control, Gap phenomena. 2010 MSC: 49N25, 34K45, 49K15.

\section{Introduction}

It is well known in Optimization Theory that the infimum cost is 'stable' under structural changes, if the optimization problem considered is normal, i.e. Lagrange multiplier rules are valid in a form that requires the cost multiplier to differ from zero (see, e.g., [6], [14]). Likewise in Optimal Control Theory, it has been shown that, under normality-type hypotheses, the infimum cost will not decrease when we enlarge the class of state trajectories to include relaxed state trajectories, i.e. trajectories whose derivatives lie in the convexified velocity set, a procedure that can be interpreted as a (infinite-dimensional) structural change to the domain of the optimal control problem under consideration. When there is no such decrease, we say 'there is no infimum gap' (see [33], [32], 28], [29]). We remark that no infimum gap can occur when the right endpoint of state trajectories is free, in consequence of the Relaxation Theorem which tells us that the set of state trajectories is ' $C^{0}$-dense' in the set of relaxed state trajectories. But infimum gaps may arise when the right endpoint is constrained.

Similar considerations come into play in connection with the following class of optimal control problems, in which the original dynamics are unbounded and the customary coercivity hypotheses, which have the effect of excluding optimal trajectories that are discontinuous, are no longer invoked.

$$
(P)\left\{\begin{array}{l}
\text { Minimize } h\left(t_{1}, x\left(t_{1}\right), t_{2}, x\left(t_{2}\right), v\left(t_{2}\right)\right) \\
\text { over } t_{1}, t_{2} \in \mathbb{R}, t_{1}<t_{2},(x, v, u) \in W^{1,1}\left(\left[t_{1}, t_{2}\right] ; \mathbb{R}^{n} \times \mathbb{R} \times \mathbb{R}^{m}\right) \text { satisfying } \\
\frac{d x}{d t}(t)=f(t, x(t))+\sum_{j=1}^{m} g_{j}(t, x(t)) \frac{d u^{j}}{d t}(t) \quad \text { a.e. } t \in\left[t_{1}, t_{2}\right] \\
\frac{d v}{d t}(t)=\left|\frac{d u}{d t}(t)\right| \quad \text { a.e. } t \in\left[t_{1}, t_{2}\right] \\
\frac{d u}{d t}(t) \in \mathcal{C} \quad \text { a.e. } t \in\left[t_{1}, t_{2}\right], \\
v\left(t_{1}\right)=0, \quad v\left(t_{2}\right) \leq K, \quad\left(t_{1}, x\left(t_{1}\right), t_{2}, x\left(t_{2}\right)\right) \in \mathcal{T},
\end{array}\right.
$$

where $K>0$ is a fixed constant (possibly equal to $+\infty$ ), $\mathcal{C} \subseteq \mathbb{R}^{m}$ is a closed convex cone, and the end-point constraint $\mathcal{T} \subseteq \mathbb{R} \times \mathbb{R}^{n} \times \mathbb{R} \times \mathbb{R}^{n}$ is a closed subset. Notice that for every $t \in\left[t_{1}, t_{2}\right], v(t)$ is nothing but the total variation of $u$ on $\left[t_{1}, t\right]$. In particular, the choice $K=+\infty$ means that there are no constraints on the total variation of the controls $u$. Since the derivatives $\frac{d u}{d t}$ - here playing the role of controls - are not $L^{\infty}$ uniformly bounded, in general, due to a lack of coerciveness, minima do not exist in the set of $W^{1,1}$ trajectories, even if one assumes the control cone $\mathcal{C}$ to be convex. So, in general, minimizing sequences of trajectories do not even converge to a continuous path. This is why we call these problems impulsive. We remark that the occurrence of impulses (i.e. discontinuities of $(x, u, v)(\cdot)$ ) is not ruled out by the weak coerciveness assumption $K<+\infty$. However, through a timereparameterization $t=t(s)$ based on the total variation map $v(\cdot)$ one can reformulate problem 
(P) as the following equivalent space-time minimum control problem, having the same form but with bounded control derivatives.

$$
\left(P_{e}\right)\left\{\begin{array}{l}
\text { Minimize } h\left(y^{0}(0), y(0), y^{0}(S), y(S), \nu(S)\right) \\
\text { over } S>0,\left(y^{0}, y, \nu, \varphi^{0}, \varphi\right) \in W^{1,1}\left([0, S] ; \mathbb{R} \times \mathbb{R}^{n} \times \mathbb{R} \times \mathbb{R} \times \mathbb{R}^{m}\right) \text { satisfying } \\
\frac{d y^{0}}{d s}(s)=\frac{d \varphi^{0}}{d s}(s) \text { a.e. } s \in[0, S] \\
\frac{d y}{d s}(s)=f\left(y^{0}(s), y(s)\right) \frac{d \varphi^{0}}{d s}(s)+\sum_{j=1}^{m} g_{j}\left(y^{0}(s), y(s)\right) \frac{d \varphi^{j}}{d s}(s) \text { a.e. } s \in[0, S], \\
\frac{d \nu}{d s}(s)=\left|\frac{d \varphi}{d s}(s)\right| \quad \text { a.e. } s \in[0, S], \\
\left(\frac{d \varphi^{0}}{d s}, \frac{d \varphi}{d s}\right)(s) \in \mathbf{C} \text { a.e. } s \in[0, S], \\
\nu(0)=0, \quad \nu(S) \leq K, \quad\left(y^{0}(0), y(0), y^{0}(S), y(S)\right) \in \mathcal{T},
\end{array}\right.
$$

where

$$
\mathbf{C}:=\left\{\left(w^{0}, w\right) \in \mathbb{R}_{+} \times \mathcal{C}: w^{0}+|w|=1\right\}
$$

and $\varphi^{0}:[0, S] \rightarrow\left[t_{1}, t_{2}\right]$ is a surjective, non-decreasing, parameterization of $\left[t_{1}, t_{2}\right]$. The controls $\left(\varphi^{0}, \varphi\right)$ verify $\frac{d \varphi^{0}}{d s}(s)+\left|\frac{d \varphi}{d s}(s)\right|=1$ a.e., so they are 1-Lipschitz continuous. The embedding of the problem (1) into the standard control problem (2) is obtained by setting

$$
s(t):=\int_{t_{1}}^{t}\left(1+\left|\frac{d u}{d \tau}(\tau)\right|\right) d \tau \quad\left(\varphi^{0}, \varphi\right):=(i d, u) \circ s^{-1} \quad\left(y^{0}, y\right):=(i d, x) \circ s^{-1}
$$

Let us point out that by allowing the time-maps $\varphi^{0}$ to be constant on non-degenerate intervals 1, we arrive at parameterized limits of trajectory graphs -here called extended sense trajectories - which are not graphs of trajectories of the original problem. For a selective bibliography on this subject, we refer the reader to [7], [20], [21], [22], 223], 19], [25], [18], [16], [30], 34] [3], 4], 26], 19] and to the references therein. In particular, the state trajectories $y$ may happen to be non-constant on $s$-subintervals where the real time $t=y^{0}$ is constant. On the other hand, it is known that $s \mapsto\left(\varphi^{0}, \varphi\right)(s)$ coincides with the reparameterization $s \mapsto(i d, u)(t(s))$ of the graph $t \mapsto(i d, u)(t)$ of some strict sense-i.e. absolutely continuouscontrol $u$ if and only if $\frac{d \varphi^{0}}{d s}>0$ almost everywhere.

Since the set of embedded strict sense trajectories 2 is $C^{0}$-dense in the set of extended sense trajectories, it is natural to address the infimum gap issue, as has previously been done in the context of relaxation for optimal control problems ([33], [32], 28], [29]). That is, one can question whether the infimum value of the functional among the strict-sense trajectories happens to be greater than the corresponding infimum among the extended-sense trajectories.

\footnotetext{
${ }^{1} \mathrm{~A}$ distributional, or measure-theoretical, approach is out of question here, in view of the fact that the vector fields $g_{i}$ are allowed to be non-commutative, see [7] [17].

${ }^{2}$ Namely, the extended sense trajectories associated to graph reparameterizations of strict-sense controls.
} 
Such infimum gaps may actually occur, as shown by simple examples. In the present paper we aim to explore the connection between 'normality' of a minimizer of problem $\left(P_{e}\right)$ and the occurrence of an infimum gap. In this case, normality means that if $\left(p_{0}, p, \pi, \lambda\right)$ is an arbitrary set of 'multipliers' ( i.e., an adjoint path) appearing in the Maximum Principle 3 , then $\lambda>0$. Omitting some details concerning the precise hypotheses we impose on the data, our main result (see Theorem 4.1) may be summarized as follows:

Theorem 1.1. Consider the optimal control problem (11) and its extended sense formulation (2). Assume that some minimizer for $\left(P_{e}\right)$ is a normal extremal. Then there is no infimum gap.

As we shall see, this relation is proved by means of a topological argument concerning the properties of so-called isolated extended sense feasible trajectories (see Theorem 4.2). We point out that our normality hypotheses are of more theoretical than practical interest. Indeed to check them requires knowledge of all sets of Lagrange multipliers $\left(p_{0}, p, \pi, \lambda\right)$ in the Maximum Principle, associated with some minimizer. Yet, this drawback may be overcome in certain situations in which verifiable condition on the data can be identified, guaranteeing that every set of multipliers is normal. Such considerations are the motivation for the last part of the paper (see Section 5), where several sufficient conditions for normality are provided in terms of quite reasonable assumptions on the end-point constraints and the vector fields $f, g_{1}, \ldots, g_{m}$.

A 'no gap condition' is clearly desirable, in particular when numerical schemes are employed to solve specific problems. We point out that consideration of impulsive systems is crucial in many applications (see, e.g. 5], 12], 15]). Instances in mechanics are situations where some state parameters $\left(u_{1}, \ldots, u_{m}\right)$ are regarded as controls $([10],[11],[9])$. The fact that the derivatives of these controls appear linearly in the dynamical equations (rather than quadratically) is an intrinsic, metric property of the foliation $u=c, c \in \mathbb{R}^{m}$, when the space of states is endowed with the kinetic energy metric. Examples where this property is verified include a swing where the length of the swing is regarded as the control input, and a multiple pendulum where the control inputs are identified with the mutual angles between adjacent single pendulums (see e.g. [31], [8]).

The paper is organized as follows: in Section 2 we describe how the original problem is embedded in the extended problem; Section 3 concerns a Maximum Principle for the extended problem, which, compared with the standard version, includes an improved non-triviality condition. In Section 4 we prove that an isolated extended-sense extremal cannot be normal and, as a corollary, we deduce the main result. In Section 5 we identify some classes of problems where normality -hence the non occurrence of gap-phenomena- can be established a priori, without any knowledge of the multipliers associated with the given extended-sense minimizer, as earlier anticipated. Finally, in Section 6, we present some instructive examples -including one where normality is shown to be not necessary to rule out gap phenomena.

Preliminaries and notation. In an Euclidean space of dimension $N$, the norm $|x|$ of a

\footnotetext{
${ }^{3}$ The adjoint component $p_{0}$ corresponds to the time variable $y^{0}$, while $\pi$ corresponds to the variable 'total variation', $\nu$ (see below).
} 
vector $x$ is defined as

$$
|x|:=\left(\sum_{i=1}^{N}\left(x^{i}\right)^{2}\right)^{1 / 2}
$$

and the closed unit ball $\{x|| x \mid \leq 1\}$ is denoted by $\mathbb{B}_{N}$. The surface of the unit ball $\{x|| x \mid=1\}$ is written $\partial \mathbb{B}_{N} . \mathbb{R}^{+}:=[0,+\infty)$. $d_{D}(x)$ denotes the Euclidean distance of the point $x \in E$ from a given closed subset $D \subset E$, namely $d_{D}(x):=\min \left\{\left|x-x^{\prime}\right| \mid x^{\prime} \in D\right\}$.

Some standard constructs from nonsmooth analysis are employed in this paper. Background material may be found in a number of texts, examples of which include [13], [24] and [27].

Definition 1.1. Take a closed set $D \subset \mathbb{R}^{k}$ and a point $\bar{x} \in D$. The limiting normal cone $N_{D}(\bar{x})$ of $D$ at $\bar{x}$ is defined to be

$$
N_{D}(\bar{x}):=\left\{p \mid \exists x_{i} \stackrel{D}{\longrightarrow} \bar{x}, p_{i} \longrightarrow p \text { s.t. } \limsup _{\substack{D \\ x \rightarrow x_{i}}} \frac{p_{i} \cdot\left(x-x_{i}\right)}{\left|x-x_{i}\right|} \leq 0 \text { for each } i\right\},
$$

in which $x_{i} \stackrel{D}{\longrightarrow} \bar{x}$ is notation conveying the information ' $x_{i} \rightarrow \bar{x}$ ' and ' $x_{i} \in D$ for all $i$ '.

Definition 1.2. Take a lower semicontinuous function $f: \mathbb{R}^{k} \rightarrow \mathbb{R}$ and a point $\bar{x} \in \mathbb{R}^{k}$. The limiting subdifferential of $f$ at $\bar{x}$ is

$$
\partial f(\bar{x})=\left\{\xi \mid \exists \xi_{i} \rightarrow \xi \text { and } x_{i} \rightarrow \bar{x} \text { s.t. } \limsup _{x \rightarrow x_{i}} \frac{\xi_{i} \cdot\left(x-x_{i}\right)-f(x)+f\left(x_{i}\right)}{\left|x-x_{i}\right|} \leq 0 \text { for each } i\right\} .
$$

We take note, for future use, of the following facts about the limiting subgradient of the distance function $d_{D}$ from an arbitrary closed set $D$ (see, e.g., [27, Section 4.8]):

i) if $\bar{x} \notin D$ and $\xi \in \partial d_{D}(\bar{x})$ then $|\xi|=1$;

ii) $\partial d_{D}(\bar{x})=N_{D}(\bar{x}) \cap \mathbb{B}_{k}$ whenever $\bar{x} \in D$.

\section{The Optimal Control Problem and its Impulsive Extension}

\subsection{The optimal control problem}

Fix $K \in(0,+\infty]$, a closed, convex cone $\mathcal{C} \subseteq \mathbb{R}^{m}$ and a closed set $\mathcal{T} \subseteq \mathbb{R} \times \mathbb{R}^{n} \times \mathbb{R} \times \mathbb{R}^{n}$, and consider the optimal control problem $(P)$ formulated in the Introduction. We shall invoke the following hypothesis

(H1): (i) the vector fields $f: \mathbb{R} \times \mathbb{R}^{n} \rightarrow \mathbb{R}^{n}, g_{j}: \mathbb{R} \times \mathbb{R}^{n} \rightarrow \mathbb{R}^{n}, j=1, \ldots, m$, are of class $C^{1}$ and for some $A, B>0$, verify

$$
|f(t, x)|+\left|g_{1}(t, x)\right|+\cdots+\left|g_{m}(t, x)\right| \leq A|x|+B \quad \forall(t, x) \in \mathbb{R} \times \mathbb{R}^{n} ;
$$

(ii) the function $h: \mathbb{R} \times \mathbb{R}^{n} \times \mathbb{R} \times \mathbb{R}^{n} \times \mathbb{R} \rightarrow \mathbb{R}$ is of class $C^{1}$ and for every $\left(t_{1}, x_{1}, t_{2}, x_{2}\right)$, the map $v \mapsto h\left(t_{1}, x_{1}, t_{2}, x_{2}, v\right)$ is monotone non-decreasing.

Remark 2.1. By means of the addition of the trivial equations $\frac{d \tilde{x}}{d t}(t)=\frac{d u}{d t}(t)$, where $\tilde{x}=$ $\left(x^{n+1}, \ldots, x^{n+m}\right)$, we can allow $h, f, g_{j}, j=1, \ldots, m$ to depend on $u$ as well as on $(t, x)$. 
Definition 2.1 (Strict sense processes). Let $t_{1}, t_{2} \in \mathbb{R}$ verify $t_{1}<t_{2}$. We call a function $u \in W^{1,1}\left(\left[t_{1}, t_{2}\right] ; \mathbb{R}^{m}\right)$ a strict sense control on $\left[t_{1}, t_{2}\right]$ if $\frac{d u}{d t}(t) \in \mathcal{C}$ a.e. in $\left[t_{1}, t_{2}\right]$. A strict sense process is a five-tuple $\left(t_{1}, t_{2}, x, v, u\right), t_{1}<t_{2}$, in which $u$ is a strict sense control on $\left[t_{1}, t_{2}\right]$ and $(x, v)$ is a $W^{1,1}\left(\left[t_{1}, t_{2}\right] ; \mathbb{R}^{n} \times \mathbb{R}\right)$ function satisfying

$$
\left\{\begin{array}{l}
\frac{d x}{d t}(t)=f(t, x(t))+\sum_{j=1}^{m} g_{j}(t, x(t)) \frac{d u^{j}}{d t}(t) \quad \text { a.e. } t \in\left[t_{1}, t_{2}\right] \\
\frac{d v}{d t}(t)=\left|\frac{d u}{d t}(t)\right| \quad \text { a.e. } t \in\left[t_{1}, t_{2}\right] .
\end{array}\right.
$$

If $\left(t_{1}, t_{2}, x, v, u\right)$ is a strict sense process, the four-tuple $\left(t_{1}, t_{2}, x, v\right) \in \mathbb{R}^{2} \times W^{1,1}\left(\left[t_{1}, t_{2}\right] ; \mathbb{R}^{n} \times \mathbb{R}\right)$ is called the (strict sense) trajectory corresponding to $\left(t_{1}, t_{2}, x, v, u\right)$. If a strict sense process $\left(t_{1}, t_{2}, x, v, u\right)$ also satisfies $v\left(t_{1}\right)=0, v\left(t_{2}\right) \leq K$ and $\left(t_{1}, x\left(t_{1}\right), t_{2}, x\left(t_{2}\right)\right) \in \mathcal{T}$, we say it is feasible.

Let us observe that there is a trivial one to one correspondence between trajectories $\left(t_{1}, t_{2}, x, v\right)$ and the four-tuple one obtains by extending continuously $(x, v)$ to $\mathbb{R}$ in such a way that both of them are constant outside the original domain $\left[t_{1}, t_{2}\right]$. In order to define a metric on strict sense trajectories we shall always consider their extension to $\mathbb{R}$. Let us define the distance

$$
d_{\infty}\left(\left(t_{1}, t_{2}, x, v\right),\left(\bar{t}_{1}, \bar{t}_{2}, \bar{x}, \bar{v}\right)\right):=\left|t_{1}-\bar{t}_{1}\right|+\left|t_{2}-\bar{t}_{2}\right|+\|(x, v)-(\bar{x}, \bar{v})\|_{L^{\infty}(\mathbb{R})} .
$$

Definition 2.2 (Local and global strict sense minimizers). We say a feasible strict sense process $\left(\bar{t}_{1}, \bar{t}_{2}, \bar{x}, \bar{v}, \bar{u}\right)$ is a strict sense $L^{\infty}$ local minimizer if there exists $\delta>0$ such that

$$
h\left(\bar{t}_{1}, \bar{x}\left(\bar{t}_{1}\right), \bar{t}_{2}, \bar{x}\left(\bar{t}_{2}\right), \bar{v}\left(\bar{t}_{2}\right)\right) \leq h\left(t_{1}, x\left(t_{1}\right), t_{2}, x\left(t_{2}\right), v\left(t_{2}\right)\right)
$$

for all feasible strict sense processes $\left(t_{1}, t_{2}, x, v, u\right)$ verifying

$$
d_{\infty}\left(\left(t_{1}, t_{2}, x, v\right),\left(\bar{t}_{1}, \bar{t}_{2}, \bar{x}, \bar{v}\right)\right) \leq \delta
$$

If relation (6) is satisfied for all strict sense feasible processes $\left(t_{1}, t_{2}, x, v, u\right)$, we say that $\left(\bar{t}_{1}, \bar{t}_{2}, \bar{x}, \bar{v}, \bar{u}\right)$ is a strict sense $L^{\infty}$ (global) minimizer.

\subsection{The extended system}

Let $\left(t_{1}, t_{2}, x, v, u\right)$ be a strict sense process. If we reparameterize time in the graph-equation

$$
\left\{\begin{array}{l}
\frac{d x^{0}}{d t}(t)=1 \\
\frac{d x}{d t}(t)=f\left(x^{0}(t), x(t)\right)+\sum_{j=1}^{m} g_{j}\left(x^{0}(t), x(t)\right) \frac{d u^{j}}{d t}(t) \quad \text { a.e. } t \in\left[t_{1}, t_{2}\right] \\
\frac{d v}{d t}(t)=\left|\frac{d u}{d t}(t)\right| \quad \text { a.e. } t \in\left[t_{1}, t_{2}\right], \\
x^{0}\left(t_{1}\right)=t_{1},
\end{array}\right.
$$


through a bi-Lipschitz increasing, surjective map $\varphi^{0}:[0, S] \rightarrow\left[t_{1}, t_{2}\right]$, we obtain the equivalent differential system on $[0, S]$

$$
\left\{\begin{array}{l}
\frac{d y^{0}}{d s}(s)=\frac{d \varphi^{0}}{d s}(s) \text { a.e. } s \in[0, S] \\
\frac{d y}{d s}(s)=f\left(y^{0}(s), y(s)\right) \frac{d \varphi^{0}}{d s}(s)+\sum_{j=1}^{m} g_{j}\left(y^{0}(s), y(s)\right) \frac{d \varphi^{j}}{d s}(s) \text { a.e. } s \in[0, S] \\
\frac{d \nu}{d s}(s)=\left|\frac{d \varphi}{d s}(s)\right| \quad \text { a.e. } s \in[0, S] \\
\left(\frac{d \varphi^{0}}{d s}, \frac{d \varphi}{d s}\right)(s) \in \mathbf{C} \text { a.e. } s \in[0, S]
\end{array}\right.
$$

where

$$
\mathbf{C}:=\left\{\left(w^{0}, w\right) \in \mathbb{R}_{+} \times \mathcal{C}: w^{0}+|w|=1\right\} .
$$

If, however, we allow the map $\varphi^{0}$ merely to be non-decreasing, we arrive at a new, impulsive, system, in which the $s$-intervals where $\varphi^{0}$ is constant represent the arcs of (nonlinear) instantaneous evolution of both the control and the state:

Definition 2.3 (Extended Sense Processes). An extended sense process $\left(S, y^{0}, y, \nu, \varphi^{0}, \varphi\right)$ comprises $S \geq 0$ and Lipschitz continuous functions $\left(y^{0}, y, \nu, \varphi^{0}, \varphi\right):[0, S] \rightarrow \mathbb{R} \times \mathbb{R}^{n} \times \mathbb{R} \times$ $\mathbb{R} \times \mathbb{R}^{m}$ satisfying (8) .

Remark 2.2. We observe that system (8) is rate independent. Indeed, if one considers a bi-Lipschitz increasing map $\sigma:[0, S] \rightarrow[0, \tilde{S}]$, then $\left(y^{0}, y, \nu\right) \circ \sigma^{-1}$ is a solution of (8) on $[0, \tilde{S}]$ corresponding to the control $\left(\varphi^{0}, \varphi\right) \circ \sigma^{-1}$ if and only if $\left(y^{0}, y, \nu\right)$ is a solution of (8) on $[0, S]$ corresponding to the control $\left(\varphi^{0}, \varphi\right)$. Thus, imposing the condition on controls

$$
\frac{d \varphi^{0}}{d s}(s)+\left|\frac{d \varphi}{d s}(s)\right|=1 \quad \text { a.e. } s \in[0, S]
$$

is a convenient, but arbitrary, choice 4 .

There is a natural embedding $\mathcal{I}$ of the family of strict sense processes into the family of extended sense processes, which is expressed by

$$
\mathcal{I}\left(t_{1}, t_{2}, x, v, u\right):=\left(S, y^{0}, y, \nu, \varphi^{0}, \varphi\right)
$$

where

$$
\begin{aligned}
& \sigma(t):=\int_{t_{1}}^{t}\left(1+\left|\frac{d u}{d \tau}(\tau)\right|\right) d \tau, \quad S:=\sigma\left(t_{2}\right) \\
& \left(y^{0}, y, \nu, \varphi^{0}, \varphi\right)(s):=(i d, x, v, i d, u)\left(\sigma^{-1}(s)\right) \quad \forall s \in[0, S]
\end{aligned}
$$

\footnotetext{
${ }^{4}$ For instance, one could have used also controls verifying $\left(\frac{d \varphi^{0}}{d s}, \frac{d \varphi}{d s}\right)(s) \in \mathbf{C}$ with

$\mathbf{C}:=\left\{\left(w^{0}, w\right) \in \mathbb{R}_{+} \times \mathcal{C}:\left|\left(w^{0}, w\right)\right|=1\right\} \quad$ or $\quad \mathbf{C}:=\left\{\left(w^{0}, w\right) \in \mathbb{R}_{+} \times \mathcal{C}:\left|w^{0}\right|+\left|w^{1}\right|+\ldots+\left|w^{m}\right|=1\right\}$

or even $\mathbf{C}:=\left\{\left(w^{0}, w\right) \in \mathbb{R}_{+} \times \mathcal{C}: \alpha<w^{0}+|w| \leq \beta\right\}$, with any $\alpha, \beta$ such that $0<\alpha<\beta$ (with the latter choice, different pairs may well represent the same control $u$ ).
} 
Notice, in particular, that

$$
\nu(S)=v\left(t_{2}\right) .
$$

Observe also that the map $\varphi^{0}\left(=\sigma^{-1}\right):[0, S] \rightarrow\left[t_{1}, t_{2}\right]$ is increasing and 1-Lipschitz continuous. Furthermore it verifies $\frac{d \varphi^{0}}{d s}(s)>0$ for almost every $s \in[0, S]$. Actually, such mappings provide a characterization of extended sense controls $\left(\varphi^{0}, \varphi\right)$ that are graphs of absolutely continuous, strict sense controls $u$ :

Lemma 2.1. The embedding $\mathcal{I}$ is injective. Moreover, the image space of the embedding $\mathcal{I}$ comprises the subclass of extended sense processes $\left(S, y^{0}, y, \nu, \varphi^{0}, \varphi\right)$ that satisfy $\frac{d \varphi^{0}}{d s}(s)>0$ almost everywhere. Actually, for every such extended sense process the map $\varphi^{0}$ is invertible with inverse $\left(\varphi^{0}\right)^{-1}$ absolutely continuous. The map $\mathcal{I}^{-1}$ is defined (on the image of $\mathcal{I}$ ) by

$$
\mathcal{I}^{-1}\left(S, y^{0}, y, \nu, \varphi^{0}, \varphi\right)=\left(t_{1}, t_{2}, x, v, u\right):=\left(\varphi^{0}(0), \varphi^{0}(S), y \circ\left(\varphi^{0}\right)^{-1}, \nu \circ\left(\varphi^{0}\right)^{-1}, \varphi \circ\left(\varphi^{0}\right)^{-1}\right) .
$$

See e.g. 1] for a proof of this standard result.

\subsection{The extended optimal control problem}

Consider the extended optimal control problem

$$
\left(P_{e}\right)\left\{\begin{array}{l}
\text { Minimize } h\left(y^{0}(0), y(0), y^{0}(S), y(S), \nu(S)\right) \\
\text { over } S>0,\left(y^{0}, y, \nu, \varphi^{0}, \varphi\right) \in W^{1,1}\left([0, S] ; \mathbb{R} \times \mathbb{R}^{n} \times \mathbb{R} \times \mathbb{R} \times \mathbb{R}^{m}\right) \text { satisfying } \\
\frac{d y^{0}}{d s}(s)=\frac{d \varphi^{0}}{d s}(s) \text { a.e. } s \in[0, S], \\
\frac{d y}{d s}(s)=f\left(y^{0}(s), y(s)\right) \frac{d \varphi^{0}}{d s}(s)+\sum_{j=1}^{m} g_{j}\left(y^{0}(s), y(s)\right) \frac{d \varphi^{j}}{d s}(s) \text { a.e. } s \in[0, S], \\
\frac{d \nu}{d s}(s)=\left|\frac{d \varphi}{d s}(s)\right| \quad \text { a.e. } s \in[0, S], \\
\left(\frac{d \varphi^{0}}{d s}, \frac{d \varphi}{d s}\right)(s) \in \mathbf{C} \text { a.e. } s \in[0, S], \\
\nu(0)=0, \quad \nu(S) \leq K, \quad\left(y^{0}(0), y(0), y^{0}(S), y(S)\right) \in \mathcal{T} .
\end{array}\right.
$$

Definition 2.4. We say that an extended sense process $\left(S, y^{0}, y, \nu, \varphi^{0}, \varphi\right)$ is feasible for $\left(P_{e}\right)$ if

$$
\nu(0)=0, \quad \nu(S) \leq K, \quad\left(y^{0}(0), y(0), y^{0}(S), y(S)\right) \in \mathcal{T} .
$$

Definition 2.5. A feasible extended sense process $\left(\bar{S}, \bar{y}^{0}, \bar{y}, \bar{\nu}, \bar{\varphi}^{0}, \bar{\varphi}\right)$ is said to be an extended sense $L^{\infty}$ local minimizer for $\left(P_{e}\right)$ if there exists $\delta>0$ such that :

$$
h\left(\bar{y}^{0}(0), \bar{y}(0), \bar{y}^{0}(\bar{S}), \bar{y}(\bar{S}), \bar{\nu}(\bar{S})\right) \leq h\left(y^{0}(0), y(0), y^{0}(S), y(S), \nu(S)\right)
$$

\footnotetext{
${ }^{5}$ Notice that the injectivity is a consequence of the fact that we require $\left(\varphi^{0}, \varphi\right)$ to verify $\frac{d \varphi^{0}}{d s}(s)+\left|\frac{d \varphi^{0}}{d s}(s)\right|=1$ a.e. in $[0, S]$.
} 
for all extended sense feasible processes $\left(S, y^{0}, y, \nu, \varphi^{0}, \varphi\right)$ satisfying

$$
d_{\infty}\left(\left(y^{0}(0), y^{0}(S), y, \nu\right),\left(\bar{y}^{0}(0), \bar{y}^{0}(\bar{S}), \bar{y}, \bar{\nu}\right)\right) \leq \delta \text {. } 6
$$

If (11) is satisfied for all extended sense feasible processes $\left(S, y^{0}, y, \nu, \varphi^{0}, \varphi\right)$, we say that $\left(\bar{S}, \bar{y}^{0}, \bar{y}, \bar{\nu}, \bar{\varphi}^{0}, \bar{\varphi}\right)$ is an extended sense $L^{\infty}$ (global) minimizer.

Let us remark that the notion of extended sense $L^{\infty}$ local minimizer for $\left(P_{e}\right)$ is consistent with the definition of $L^{\infty}$ local minimizer for problem $(\mathrm{P})$. To be precise, it is not difficult to prove the following result.

Lemma 2.2. A process $\left(\bar{t}_{1}, \bar{t}_{2}, \bar{x}, \bar{v}, \bar{u}\right)$ is a $L^{\infty}$ local minimizer for problem $(P)$ if and only if

$$
\left(\bar{S}, \bar{y}^{0}, \bar{y}, \bar{\nu}, \bar{\varphi}^{0}, \bar{\varphi}\right):=\mathcal{I}\left(\bar{t}_{1}, \bar{t}_{2}, \bar{x}, \bar{v}, \bar{u}\right)
$$

is an extended sense $L^{\infty}$ local minimizer for problem $\left(P_{e}\right)$ among embedded strict sense feasible processes. Moreover, these equivalent properties imply

$$
h\left(\bar{y}^{0}(0), \bar{y}(0), \bar{y}^{0}(\bar{S}), \bar{y}(\bar{S}), \bar{\nu}(\bar{S})\right)=h\left(\bar{t}_{1}, \bar{x}\left(\bar{t}_{1}\right), \bar{t}_{2}, \bar{x}\left(\bar{t}_{2}\right), \bar{v}\left(\bar{t}_{2}\right)\right) .
$$

Remark 2.3. Although the question of existence is not addressed in this paper, we point out that the existence of an optimal extended sense feasible process has been established in several cases. For instance, when either $K<+\infty$ or $h\left(t_{1}, x_{1}, t_{2}, x_{2}, v\right) \geq \psi(v)$ with $\lim _{v \rightarrow+\infty} \psi(v)=+\infty$ and the target is of the form $\mathcal{T}=\left\{\left(\bar{t}_{1}, \bar{x}_{1}, \bar{t}_{2}\right)\right\} \times \tilde{\mathcal{T}}$ for a given closed set $\tilde{\mathcal{T}} \subset \mathbb{R}^{n}$, one can establish existence by compactness and the continuity -in suitable topologies- of the input-output map7 (see, e.g., [7], [20], [19], [16]). These include so-called weakly coercive problems, namely those problems where the cost has the form $J\left(t_{1}, t_{2}, x, v, u\right)=\int_{t_{1}}^{t_{2}}\left[\ell_{0}(t, x(t))+\right.$ $\left.\ell_{1}(t, x(t))|\dot{u}(t)|\right] d t$, with $\ell_{1}(t, x) \geq C$ for some constant $C>0$.

\section{Necessary Conditions for the Extended Optimal Control Problem}

This section provides necessary conditions, in the form of a Pontryagin Maximum Principle (PMP), for extended sense $L^{\infty}$ local minimizers.

Theorem 3.1. Take an extended sense $L^{\infty}$ local minimizer for $\left(P_{e}\right),\left(\bar{S}, \bar{y}^{0}, \bar{y}, \bar{\nu}, \bar{\varphi}^{0}, \bar{\varphi}\right)$. Assume hypothesis (H1) is satisfied. Then the following conditions are verified: there exist $\left(p_{0}, p\right) \in W^{1,1}\left([0, \bar{S}] ; \mathbb{R}^{1+n}\right)$ and real numbers $\pi, \lambda$, with $\pi \leq 0$ and $\lambda \geq 0$, such that

$$
\left(p_{0}, p, \lambda\right) \neq(0,0,0)
$$

\footnotetext{
${ }^{6}$ As in the strict sense case, we mean that $(\bar{y}, \bar{\nu})$ and $(y, \nu)$ are continuously extended to $\mathbb{R}$ so that they are constant outside the original domains $[0, \bar{S}]$ and $[0, S]$, respectively. Let us observe, incidentally, that

$$
S=\varphi^{0}(S)-\varphi^{0}(0)+\nu(S)=y^{0}(S)-y^{0}(0)+\nu(S)
$$

${ }^{7}$ Of course one has to assume that the set of feasible extended sense trajectories is non-empty.
} 


$$
\begin{aligned}
& \left\{\begin{array}{l}
\frac{d p_{0}}{d s}(s)=-p(s) \cdot\left(\frac{\partial f}{\partial t}\left(\bar{y}^{0}(s), \bar{y}(s)\right) \frac{d \bar{\varphi}^{0}}{d s}(s)+\sum_{j=1}^{m} \frac{\partial g_{j}}{\partial t}\left(\bar{y}^{0}(s), \bar{y}(s)\right) \frac{d \bar{\varphi}^{j}}{d s}(s)\right) \quad \text { a.e. } s \in[0, \bar{S}], \\
\frac{d p}{d s}(s)=-p(s) \cdot\left(\frac{\partial f}{\partial x}\left(\bar{y}^{0}(s), \bar{y}(s)\right) \frac{d \bar{\varphi}^{0}}{d s}(s)+\sum_{j=1}^{m} \frac{\partial g_{j}}{\partial x}\left(\bar{y}^{0}(s), \bar{y}(s)\right) \frac{d \bar{\varphi}^{j}}{d s}(s)\right) \quad \text { a.e. } s \in[0, \bar{S}],
\end{array}\right. \\
& p(s) \cdot\left(f\left(\bar{y}^{0}(s), \bar{y}(s)\right) \frac{d \bar{\varphi}^{0}}{d s}(s)+\sum_{j=1}^{m} g_{j}\left(\bar{y}^{0}(s), \bar{y}(s)\right) \frac{d \bar{\varphi}^{j}}{d s}(s)\right)+p_{0}(s) \frac{d \bar{\varphi}^{0}}{d s}(s)+\pi\left|\frac{d \bar{\varphi}}{d s}\right|(s)= \\
& \max _{\left(w^{0}, w\right) \in \mathbf{C}}\left\{p(s) \cdot\left(f\left(\bar{y}^{0}(s), \bar{y}(s)\right) w^{0}+\sum_{j=1}^{m} g_{j}\left(\bar{y}^{0}(s), \bar{y}(s)\right) w^{j}\right)+p_{0}(s) w^{0}+\pi|w|\right\}=0
\end{aligned}
$$

a.e. $s \in[0, \bar{S}]$ and

$$
\begin{aligned}
& \left(p_{0}(0), p(0),-p_{0}(\bar{S}),-p(\bar{S}),-\pi\right) \in \\
& \quad \lambda \nabla h\left(\bar{y}^{0}(0), \bar{y}(0), \bar{y}^{0}(\bar{S}), \bar{y}(\bar{S}), \bar{\nu}(\bar{S})\right)+N_{\mathcal{T} \times[0, K]}\left(\bar{y}^{0}(0), \bar{y}(0), \bar{y}^{0}(\bar{S}), \bar{y}(\bar{S}), \bar{\nu}(\bar{S})\right) .
\end{aligned}
$$

Moreover, additional 'multiplier' information is available in the following special cases:

(i) if $\lambda \frac{\partial h}{\partial v}\left(\bar{y}^{0}(0), \bar{y}(0), \bar{y}^{0}(\bar{S}), \bar{y}(\bar{S}), \bar{\nu}(\bar{S})\right)=0$ and $\bar{\nu}(\bar{S})<K$ then

$$
\pi=0
$$

(ii) if $\bar{y}^{0}(0)<\bar{y}^{0}(\bar{S})$, inequality (13) can be strengthened to

$$
(p, \lambda) \neq(0,0)
$$

Remark 3.1. The standard PMP applied to the extended problem tells us that there exists a non-trivial multiplier set $\left(p_{0}, p, \pi, \lambda\right)$ satisfying conditions (14)-(16). The novelty of Thm. 3.1 consists in the stronger non-triviality condition (13) and the additional information concerning the multipliers non-triviality in some cases of interest.

Proof of Thm. 3.1. $\left(P_{e}\right)$ is a standard optimal control problem, to which the 'free end-time' PMP is applicable (see, e.g., [27, Thm. 8.7.1]), with reference to the $L^{\infty}$ local extended sense minimizer $\left(\bar{S}, \bar{y}^{0}, \bar{y}, \bar{\varphi}^{0}, \bar{\varphi}\right)$. This yields the existence of $\left(p_{0}, p\right) \in W^{1,1}\left([0, \bar{S}] ; \mathbb{R}^{1+n}\right)$ and $\pi \in \mathbb{R}$, $\lambda \geq 0$ satisfying (14)-(15), the transversality conditions (16) and the non-triviality condition

$$
\left(p_{0}, p, \pi, \lambda\right) \neq 0 \text {. }
$$

Since $\frac{\partial h}{\partial v}\left(\bar{y}^{0}(0), \bar{y}(0), \bar{y}^{0}(\bar{S}), \bar{y}(\bar{S}), \bar{\nu}(\bar{S})\right) \geq 0$ and $N_{[0, K]}(v)=\{0\}$ for $v<K, N_{[0, K]}(K)=$ $[0,+\infty)$, it follows from (16) that $\pi=0$ as soon as $\lambda \frac{\partial h}{\partial v}\left(\bar{y}^{0}(0), \bar{y}(0), \bar{y}^{0}(\bar{S}), \bar{y}(\bar{S}), \bar{\nu}(\bar{S})\right)=0$ and $\bar{\nu}(\bar{S})<K$, while $\pi \leq 0$ in the other cases. So the proof concerning the sign of $\pi$, and also relation (i), is complete.

Next, we show that $\left(p_{0}, p, \lambda\right) \neq(0,0,0)$. Indeed, if this were not true, it would follow from (18) that $\pi \neq 0$. But then $\bar{\nu}(\bar{S})=K$. Integrating the first equation in (15), one obtains $\pi K=0$. This is not possible, since $K>0$. 
Finally, we consider relation (ii). Suppose then that $\bar{y}^{0}(0)<\bar{y}^{0}(S)$. We must show that $(p, \lambda) \neq(0,0)$. If this were not true, we would be able to deduce from (14) and (15) that $p_{0}(\cdot)$ is a constant function and

$$
p_{0}(s) \frac{d \bar{\varphi}^{0}}{d s}(s)+\pi\left|\frac{d \bar{\varphi}}{d s}(s)\right|=\max _{\left(w^{0}, w\right) \in \mathbf{C}}\left\{p_{0}(s) w^{0}+\pi|w|\right\}=0 \text { a.e. } s \in[0, \bar{S}] .
$$

If $\pi<0$, it would follow from this relation that $p_{0}(s)=0$ for all $s \in[0, \bar{S}]$. This cannot be true since, as we have shown, $\left(p_{0}, p, \lambda\right) \neq(0,0,0)$. If, on the other hand, $\pi=0$, it would follow from the preceding relation that $p_{0}(s)<0$ a.e. $s \in[0, \bar{S}]$. But then we would have $\frac{d \bar{\varphi}^{0}}{d s}(s)=0$ a.e., which would imply

$$
\bar{y}^{0}(S)-\bar{y}^{0}(0)=\int_{0}^{\bar{S}} \frac{d \bar{\varphi}^{0}}{d s}(s) d s=0
$$

This is not possible since, as we have assumed, $\bar{y}^{0}(0)<\bar{y}^{0}(\bar{S})$. From this contradiction we deduce relation (ii).

\section{4. 'No Infimum Gap' and Normality}

Write $J\left(t_{1}, t_{2}, x, v, u\right)$ for the cost of a strict sense process $\left(t_{1}, t_{2}, x, v, u\right)$ in problem $(P)$, namely,

$$
J\left(t_{1}, t_{2}, x, v, u\right):=h\left(t_{1}, x\left(t_{1}\right), t_{2}, x\left(t_{2}\right), v\left(t_{2}\right)\right),
$$

and $J_{e}\left(S, y^{0}, y, \nu, \varphi^{0}, \varphi\right)$ for the cost of an extended sense process $\left(S, y^{0}, y, \nu, \varphi^{0}, \varphi\right)$ in problem $\left(P_{e}\right)$ :

$$
J_{e}\left(S, y^{0}, y, \nu, \varphi^{0}, \varphi\right):=h\left(y^{0}(0), y(0), y^{0}(S), y(S), \nu(S)\right) .
$$

Let us also write $\mathcal{A}$ and $\mathcal{A}_{e}$ for the class of feasible strict sense processes (in problem $(P)$ ) and for the class of feasible extended sense processes (in problem $\left(P_{e}\right)$ ), respectively.

Definition 4.1. We shall say that

(i) there is no infimum gap if

$$
\begin{aligned}
\inf \left\{J_{e}\left(S, y^{0}, y, \nu, \varphi^{0}, \varphi\right) \mid\left(S, y^{0}, y\right.\right. & \left.\left., \nu, \varphi^{0}, \varphi\right) \in \mathcal{A}_{e}\right\} \\
& =\inf \left\{J\left(t_{1}, t_{2}, x, v, u\right) \mid\left(t_{1}, t_{2}, x, v, u\right) \in \mathcal{A}\right\} .
\end{aligned}
$$

Furthermore, if $\left(\bar{S}, \bar{y}^{0}, \bar{y}, \bar{\nu}, \bar{\varphi}^{0}, \bar{\varphi}\right)$ is an extended sense $L^{\infty}$ local minimizer, we shall say that

(ii) there is no local infimum gap at $\left(\bar{S}, \bar{y}^{0}, \bar{y}, \bar{\nu}, \bar{\varphi}^{0}, \bar{\varphi}\right)$ if, for some $\delta>0$,

$$
J_{e}\left(\bar{S}, \bar{y}^{0}, \bar{y}, \bar{\nu}, \bar{\varphi}^{0}, \bar{\varphi}\right)=\inf \left\{J\left(t_{1}, t_{2}, x, v, u\right) \mid\left(t_{1}, t_{2}, x, v, u\right) \in B_{\delta}\left[\left(\bar{S}, \bar{y}^{0}, \bar{y}, \bar{\nu}, \bar{\varphi}^{0}, \bar{\varphi}\right)\right]\right\}
$$

where we have set

$$
B_{\delta}\left[\left(\bar{S}, \bar{y}^{0}, \bar{y}, \bar{\nu}, \bar{\varphi}^{0}, \bar{\varphi}\right)\right]:=\left\{\begin{array}{r}
\left(t_{1}, t_{2}, x, v, u\right) \in \mathcal{A} \mid\left(S, y^{0}, y, \nu, \varphi^{0}, \varphi\right)=\mathcal{I}\left(t_{1}, t_{2}, x, v, u\right) \\
\text { and } d_{\infty}\left(\left(\bar{y}^{0}(0), \bar{y}^{0}(\bar{S}), \bar{y}, \bar{\nu}\right),\left(y^{0}(0), y^{0}(S), y, \nu\right)\right)<\delta
\end{array}\right\} .
$$


To prove Theorem 4.1 below, it will be convenient to introduce the subset $\mathcal{A}_{e}^{+} \subset \mathcal{A}_{e}$ defined by

$$
\mathcal{A}_{e}^{+}:=\left\{\left(S, y^{0}, y, \nu, \varphi^{0}, \varphi\right) \mid\left(S, y^{0}, y, \nu, \varphi^{0}, \varphi\right)=\mathcal{I}\left(t_{1}, t_{2}, x, v, u\right) \text { and }\left(t_{1}, t_{2}, x, v, u\right) \in \mathcal{A}\right\} .
$$

Using the same notation of Def. 4.1, by Lemma 2.2 we have:

$$
\inf \left\{J\left(t_{1}, t_{2}, x, v, u\right) \mid\left(t_{1}, t_{2}, x, v, u\right) \in \mathcal{A}\right\}=\inf \left\{J_{e}\left(S, y^{0}, y, \nu, \varphi^{0}, \varphi\right) \mid\left(S, y^{0}, y, \nu, \varphi^{0}, \varphi\right) \in \mathcal{A}_{e}^{+}\right\}
$$

and

$$
\begin{aligned}
& \inf \left\{J\left(t_{1}, t_{2}, x, v, u\right) \mid\left(t_{1}, t_{2}, x, v, u\right) \in B_{\delta}\left[\left(\bar{S}, \bar{y}^{0}, \bar{y}, \bar{\nu}, \bar{\varphi}^{0}, \bar{\varphi}\right)\right]\right\} \\
& =\inf \left\{J_{e}\left(S, y^{0}, y, \nu, \varphi^{0}, \varphi\right) \mid\left(S, y^{0}, y, \nu, \varphi^{0}, \varphi\right) \in \mathcal{A}_{e}^{+}\right. \text {and } \\
& \left.d_{\infty}\left(\left(\bar{y}^{0}(0), \bar{y}^{0}(\bar{S}), \bar{y}, \bar{\nu}\right),\left(y^{0}(0), y^{0}(S), y, \nu\right)\right)<\delta\right\} .
\end{aligned}
$$

Theorem 4.1. Assume Hypothesis (H1) is satisfied.

(i) Suppose that there exists an $L^{\infty}$ minimizer for $\left(P_{e}\right)$ which is a normal extremal. Then there is no infimum gap.

(ii) Take an extended sense $L^{\infty}$ local minimizer $\left(\bar{S}, \bar{y}^{0}, \bar{y}, \bar{\nu}, \bar{\varphi}^{0}, \bar{\varphi}\right)$ for $\left(P_{e}\right)$. Suppose that $\left(\bar{S}, \bar{y}^{0}, \bar{y}, \bar{\nu}, \bar{\varphi}^{0}, \bar{\varphi}\right)$ is a normal extremal. Then there is no local infimum gap at $\left(\bar{S}, \bar{y}^{0}, \bar{y}, \bar{\nu}, \bar{\varphi}^{0}, \bar{\varphi}\right)$.

Theorem 4.1 will be proved in Subsection 4.2 as a consequence of Theorem 4.2 below, which relates 'isolated processes' and normality.

\subsection{Isolated Extended Sense Feasible Processes}

Notice that, on the one hand, the trajectory of any extended sense process $\left(\bar{S}, \bar{y}^{0}, \bar{y}, \bar{\nu}, \bar{\varphi}^{0}, \bar{\varphi}\right)$ can be approximated by the trajectory of an embedded strict sense process, that is, for any $\delta>0$ we can find an embedded strict sense process $\left(S, y^{0}, y, \nu, \varphi^{0}, \varphi\right)$ such that

$$
d_{\infty}\left(\left(\bar{y}^{0}(0), \bar{y}^{0}(\bar{S}), \bar{y}, \bar{\nu}\right),\left(y^{0}(0), y^{0}(S), y, \nu\right)\right)<\delta,
$$

where $d_{\infty}$ is the metric defined in (5). On the other hand, the presence of endpoint constraints could make such an approximation unachievable, if we keep the requirement that approximating embedded strict sense processes have to be feasible as well 8 . This is because the perturbation that changes $\left(\bar{S}, \bar{y}^{0}, \bar{y}, \bar{\nu}, \bar{\varphi}^{0}, \bar{\varphi}\right)$ into a close embedded strict sense process might violate either the end-point constraints or the total variation bound. The phenomenon is captured in the following definition (of topological nature):

Definition 4.2. (Isolated feasible extended sense processes) We say that a feasible extended sense process $\left(\bar{S}, \bar{y}^{0}, \bar{y}, \bar{\nu}, \bar{\varphi}^{0}, \bar{\varphi}\right)$ is isolated if, for some $\delta>0$, there does not exist a feasible embedded strict sense process $\left(S, y^{0}, y, \nu, \varphi^{0}, \varphi\right)$ such that (20) is satisfied.

\footnotetext{
${ }^{8}$ Actually, such a requirement seems minimal if one wishes feasible extended processes to retain the physical meaning of limits of actual -i.e. strict sense feasible-- processes.
} 
A feasible extended sense process having the properties described in this definition is called 'isolated' because the state trajectory component $\left(\bar{y}^{0}, \bar{y}, \bar{\nu}\right):[0, \bar{S}] \rightarrow \mathbb{R} \times \mathbb{R}^{n} \times \mathbb{R}$ is not in the closure (w.r.t. the metric defined by the left side of $(\underline{20})$ ) of the set of state trajectories arising from embedded strict sense processes, whose endpoints lie in the target set and satisfy the total variation constraint.

In this section we give a necessary condition for a feasible extended sense process to be isolated. The relevance of this condition, which will be explored in subsequent sections, is the insights that it will provide into possible differences between the infimum cost of the optimal control problem $(P)$ and its extension $\left(P_{e}\right)$.

Definition 4.3. (Normal and abnormal extremals) A feasible extended sense process $\left(\bar{S}, \bar{y}^{0}, \bar{y}, \bar{\nu}, \bar{\varphi}^{0}, \bar{\varphi}\right)$, is said to be an extended sense extremal if there exists a set of multipliers $\left(p_{0}, p, \pi, \lambda\right)$ such that $\left(p_{0}, p, \pi, \lambda\right)$ and $\left(\bar{S}, \bar{y}^{0}, \bar{y}, \bar{\nu}, \bar{\varphi}^{0}, \bar{\varphi}\right)$ satisfy the conditions listed in Thm. 3.1. An extended sense extremal $\left(\bar{S}, \bar{y}^{0}, \bar{y}, \bar{\nu}, \bar{\varphi}^{0}, \bar{\varphi}\right)$ is said to be normal if the only possible choices of multiplier sets $\left(p_{0}, p, \pi, \lambda\right)$ associated with it are such that $\lambda>0$. If there exists at least one set of multipliers such that $\lambda=0$, we say that the extended sense extremal is abnormal.

The following result relates isolated extended sense processes and abnormality, from which the main result (Theorem 4.1) will follow.

Theorem 4.2. If a feasible extended sense process $\left(\bar{S}, \bar{y}^{0}, \bar{y}, \bar{\nu}, \bar{\varphi}^{0}, \bar{\varphi}\right)$ is isolated, then it is an abnormal extremal for $\left(P_{e}\right)$.

Proof. We may assume, without loss of generality, that (H1) (i) is replaced by the stronger hypothesis:

(H1)*: (i) the vector fields $f: \mathbb{R} \times \mathbb{R}^{n} \rightarrow \mathbb{R}^{n}, g_{j}: \mathbb{R} \times \mathbb{R}^{n} \rightarrow \mathbb{R}^{n}, j=1, \ldots, m$ are of class $C^{1}$, Lipschitz continuous and bounded.

(If only (H1) (i) is satisfied, we consider an optimal control problem related to (P), in which $f$ and the $g_{j}$ 's are modified outside a ball in $\mathbb{R} \times \mathbb{R}^{n}$ containing Graph $\left\{\left(\bar{y}^{0}, \bar{y}\right)\right\}$ in its interior, by truncation and mollification. Since the analysis involves consideration of extended sense trajectories with graphs arbitrarily close to $\operatorname{Graph}\left\{\left(\bar{y}^{0}, \bar{y}\right)\right\}$ in the Hausdorff sense and the relations appearing in the statement of the theorem concern properties of the data 'near' $\operatorname{Graph}\left\{\left(\bar{y}^{0}, \bar{y}\right)\right\}$, it suffices to prove the assertions for only the modified problem.)

Let $\left(\bar{S}, \bar{y}^{0}, \bar{y}, \bar{\nu}, \bar{\varphi}^{0}, \bar{\varphi}\right)$ be an arbitrary isolated extended sense feasible process. Define the map $\phi: \mathbb{R} \times \mathbb{R}^{n} \times \mathbb{R} \times \mathbb{R}^{n} \times \mathbb{R} \rightarrow \mathbb{R}$, given by

$$
\phi\left(y_{0}^{0}, y_{0}, y_{1}^{0}, y_{1}, v\right):=\max \left\{d_{\mathcal{T}}\left(y_{0}^{0}, y_{0}, y_{1}^{0}, y_{1}\right),(v-K) \vee 0\right\} .
$$

Take a positive sequence $\left(\epsilon_{i}\right)$ such that $\epsilon_{i} \searrow 0$. For each $i \in \mathbb{N}$, let us consider the free 
end-point optimal control problem

$$
\left(\hat{P}_{i}\right)\left\{\begin{array}{l}
\text { Minimize } \phi\left(y^{0}(0), y(0), y^{0}(\bar{S}), y(\bar{S}), \nu(\bar{S})\right) \\
\text { over }\left(y^{0}, y, \nu\right) \in W^{1,1}\left([0, \bar{S}] ; \mathbb{R} \times \mathbb{R}^{n} \times \mathbb{R}\right) \text { and measurable functions } d, w \text { satisfying } \\
\frac{d y^{0}}{d s}(s)=(1+d(s))(1-|w(s)|) \text { a.e. } s \in[0, \bar{S}], \\
\frac{d y}{d s}(s)=(1+d(s))\left(f\left(y^{0}(s), y(s)\right)(1-|w(s)|)+\sum_{j=1}^{m} g_{j}\left(y^{0}(s), y(s)\right) w^{j}(s)\right) \text { a.e. } s \in[0, \bar{S}], \\
\frac{d \nu}{d s}(s)=(1+d(s))|w(s)|, \text { a.e. } s \in[0, \bar{S}], \\
w(s) \in\left(1-\epsilon_{i}\right)\left(\mathcal{C} \cap \mathbb{B}_{m}\right), \text { a.e. } s \in[0, \bar{S}], \\
d(s) \in[-0.5,+0.5], \text { a.e. } s \in[0, \bar{S}], \\
k(0)=0
\end{array}\right.
$$

We call a collection of functions $\left(y^{0}, y, \nu, d, w\right)$ satisfying the constraints in this problem a feasible process for problem $\left(\hat{P}_{i}\right)$. (Notice that, to simplify the notation, we use here $w$ for the $s$-derivative of $\varphi$, and express the variable $\left(w^{0}, w\right)$ satisfying the constraint $\left(w^{0}, w\right) \in \mathbf{C}$, as $(1-|w|,|w|)$ where $\left.w \in\left(1-\epsilon_{i}\right)\left(\mathcal{C} \cap \mathbb{B}_{m}\right)\right)$.

For every $i \in \mathbb{N}$, let $\left(\bar{S}, \hat{y}_{i}^{0}, \hat{y}_{i}, \hat{\nu}_{i}, \hat{\varphi}_{i}^{0}, \hat{\varphi}_{i}\right)$ be the extended sense process in which

$$
\left(\hat{y}_{i}^{0}, \hat{y}_{i}, \hat{\nu}_{i}, \hat{\varphi}_{i}^{0}, \hat{\varphi}_{i}\right)(0)=\left(\bar{y}^{0}, \bar{y}, \bar{\nu}, \bar{\varphi}^{0}, \bar{\varphi}\right)(0)
$$

and, for a.e. $s \in[0, \bar{S}]$,

$$
\left(\frac{d \hat{\varphi}_{i}^{0}}{d s}, \frac{d \hat{\varphi}_{i}}{d s}\right)(s):=\left\{\begin{array}{l}
\left(\epsilon_{i},\left(1-\epsilon_{i}\right) \frac{d \bar{\varphi}}{d s}(s) /\left|\frac{d \bar{\varphi}}{d s}(s)\right|\right) \text { if } \frac{d \bar{\varphi}^{0}}{d s}(s)<\epsilon_{i}, \\
\left(\frac{d \bar{\varphi}^{0}}{d s}(s), \frac{d \bar{\varphi}}{d s}(s)\right) \text { if } \frac{d \bar{\varphi}^{0}}{d s}(s) \geq \epsilon_{i} .
\end{array}\right.
$$

Notice that for each $i \in \mathbb{N}$, on the one hand, $\frac{d \hat{\varphi}_{i}}{d s}(s) \in\left(1-\epsilon_{i}\right)\left(\mathcal{C} \cap \mathbb{B}_{m}\right)$ a.e. $s \in[0, \bar{S}]$, so that $\left(\hat{y}_{i}^{0}, \hat{y}_{i}, \hat{\nu}_{i}, 0, \frac{d \hat{\varphi}_{i}}{d s}\right)$ is a feasible process for problem $\left(\hat{P}_{i}\right)$; on the other hand, $\frac{d \hat{\varphi}_{i}^{0}}{d s}(s) \geq \epsilon_{i}>0$ a.e. $s \in[0 \bar{S}]$, so that $\left(\bar{S}, \hat{y}_{i}^{0}, \hat{y}_{i}, \hat{\nu}_{i}, \hat{\varphi}_{i}^{0}, \hat{\varphi}_{i}\right)$ is an embedded strict sense process for problem $\left(P_{e}\right)$. Moreover,

$$
\left\|\left(\frac{d \hat{\varphi}_{i}^{0}}{d s}, \frac{d \hat{\varphi}_{i}}{d s}\right)-\left(\frac{d \bar{\varphi}^{0}}{d s}, \frac{d \bar{\varphi}}{d s}\right)\right\|_{L^{\infty}(0, \bar{S})} \rightarrow 0 \text { as } i \rightarrow \infty,
$$

which implies that the $\left(\hat{\varphi}_{i}^{0}, \hat{\varphi}_{i}\right)$ converge uniformly to $\left(\bar{\varphi}^{0}, \bar{\varphi}\right)$. Noting Hypothesis (H1)* (i) and also the continuity properties of the input-output map, proved e.g.in [20], we obtain:

$$
\left\|\left(\hat{y}_{i}^{0}, \hat{y}_{i}, \hat{\nu}_{i}\right)-\left(\bar{y}^{0}, \bar{y}, \bar{\nu}\right)\right\|_{L^{\infty}(0, \bar{S})} \rightarrow 0, \text { as } i \rightarrow \infty .
$$

Since $\phi$ is non-negative valued and vanishes at $\left(\bar{y}^{0}(0), \bar{y}(0), \bar{y}^{0}(\bar{S}), \bar{y}(\bar{S}), \bar{\nu}(\bar{S})\right)$, and in view of (23), there exists a sequence $\rho_{i} \searrow 0$ such that, for each $i,\left(\hat{y}_{i}^{0}, \hat{y}_{i}, \hat{\nu}_{i}, 0, \frac{d \hat{\varphi}_{i}}{d s}\right)$ is a $\rho_{i}^{2}$-minimizer 
for $\left(\hat{P}_{i}\right)$, i.e. is a process whose cost exceeds the infimum cost for $\left(\hat{P}_{i}\right)$ by an amount not greater that $\rho_{i}^{2}$.

Problem $\left(\hat{P}_{i}\right)$ can be regarded as an optimization problem with continuous cost over elements $\left(d, w, y^{0}(0), y(0)\right)$ in a closed subset of $L^{1}([0, \bar{S}] ; \mathbb{R}) \times L^{1}\left([0, \bar{S}] ; \mathbb{R}^{m}\right) \times \mathbb{R} \times \mathbb{R}^{n}$. In consequence of Ekeland's Principle [27, Thm. 3.3.1], there exists, for each $i$, a feasible process for $\left(\hat{P}_{i}\right)$, $\left(y_{i}^{0}, y_{i}, \nu_{i}, d_{i}, w_{i}\right)$, such that

$$
\left|\left(\hat{y}_{i}^{0}, \hat{y}_{i}\right)(0)-\left(y_{i}^{0}, y_{i}\right)(0)\right| \rightarrow 0,\left\|\hat{w}_{i}-w_{i}\right\|_{L^{1}(0, \bar{S})} \rightarrow 0 \text { and }\left\|d_{i}\right\|_{L^{1}(0, \bar{S})} \rightarrow 0 \text { as } i \rightarrow \infty,
$$

and $\left(y_{i}^{0}, y_{i}, \nu_{i}, d_{i}, w_{i}\right)$ is a minimizer for

$$
\left(P_{i}\right)\left\{\begin{array}{l}
\text { Minimize } \phi\left(y^{0}(0), y(0), y^{0}(\bar{S}), y(\bar{S}), \nu(\bar{S})\right)+ \\
\quad \rho_{i} \cdot\left(\left|\left(y^{0}, y\right)(0)-\left(y_{i}^{0}, y_{i}\right)(0)\right|+\int_{0}^{\bar{S}}\left|w(s)-w_{i}(s)\right|+\left|d(s)-d_{i}(s)\right| d s\right) \\
\text { over }\left(y^{0}, y, \nu\right) \in W^{1,1}\left([0, \bar{S}] ; \mathbb{R} \times \mathbb{R}^{n} \times \mathbb{R}\right) \text { and measurable functions } \\
d:[0, \bar{S}] \rightarrow \mathbb{R}, w:[0, \bar{S}] \rightarrow \mathbb{R}^{m} \text { satisfying } \\
\frac{d y^{0}}{d s}(s)=(1+d(s))(1-|w(s)|) \text { a.e. } s \in[0, \bar{S}], \\
\frac{d y}{d s}(s)=(1+d(s))\left(f\left(y^{0}(s), y(s)\right)(1-\mid w(s))+\sum_{j=1}^{m} g_{j}\left(y^{0}(s), y(s)\right) w^{j}(s)\right) \text { a.e. } s \in[0, \bar{S}], \\
\frac{d \nu}{d s}(s)=(1+d(s))|w(s)| \\
w(s) \in\left(1-\epsilon_{i}\right)\left(\mathcal{C} \cap \mathbb{B}_{m}\right), \text { a.e. } s \in[0, \bar{S}], \\
d(s) \in[-0.5,+0.5] \text { a.e. } s \in[0, \bar{S}], \\
\nu(0)=0 .
\end{array}\right.
$$

It follows from (22) and (24) that

$$
\left\|w_{i}-\frac{d \bar{\varphi}}{d s}\right\|_{L^{1}(0, \bar{S})} \rightarrow 0 \text { and }\left\|\left(y_{i}^{0}, y_{i}, \nu_{i}\right)-\left(\bar{y}^{0}, \bar{y}, \bar{\nu}\right)\right\|_{L^{\infty}(0, \bar{S})} \rightarrow 0, \text { as } i \rightarrow \infty \text {. }
$$

By extracting subsequences, we can arrange that

$$
w_{i}(s) \rightarrow \frac{d \bar{\varphi}}{d s}(s) \text { and } d_{i}(s) \rightarrow 0 \quad \text { a.e. } s \in[0, \bar{S}] \text {, as } i \rightarrow \infty
$$

Now, apply the PMP to $\left(P_{i}\right)$ with reference to the minimizer $\left(y_{i}^{0}, y_{i}, \nu_{i}, d_{i}, w_{i}\right)$. This yields $\left(p_{0 i}, p_{i}\right) \in W^{1,1}\left([0, \bar{S}] ; \mathbb{R}^{n}\right)$ and $\pi_{i} \in \mathbb{R}$ such that the adjoint equations

$$
\begin{aligned}
& \frac{d p_{0 i}}{d s}(s)=-p_{i}(s) \cdot\left(\frac{\partial f}{\partial t}\left(y_{i}^{0}(s), y_{i}(s)\right)\left(1-\left|w_{i}(s)\right|\right)+\sum_{j=1}^{m} \frac{\partial g_{j}}{\partial t}\left(y_{i}^{0}(s), y_{i}(s)\right) w_{i}^{j}(s)\right)\left(1+d_{i}(s)\right),(27) \\
& \frac{d p_{i}}{d s}(s)=-p_{i}(s) \cdot\left(\frac{\partial f}{\partial x}\left(y_{i}^{0}(s), y_{i}(s)\right)\left(1-\left|w_{i}(s)\right|\right)+\sum_{j=1}^{m} \frac{\partial g_{j}}{\partial x}\left(y_{i}^{0}(s), y_{i}(s)\right) w_{i}^{j}(s)\right)\left(1+d_{i}(s)\right)
\end{aligned}
$$


are verified (on $[0, \bar{S}]$ ), inequality

$$
\begin{gathered}
\int_{0}^{\bar{S}}\left\{\left[p_{i}(s) \cdot\left(f\left(y_{i}^{0}(s), y_{i}(s)\right)(1-|w(s)|)+\sum_{j=1}^{m} g_{j}\left(y_{i}^{0}(s), y_{i}(s)\right) w^{j}(s)\right)\right.\right. \\
\left.\left.+p_{0 i}(s)(1-|w(s)|)+\pi_{i}|w(s)|\right](1+d(s))+\rho_{i}\left(\left|w(s)-w_{i}(s)\right|+\left|d(s)-d_{i}(s)\right|\right)\right\} d s \\
\leq \int_{0}^{\bar{S}}\left\{\left[p_{i}(s) \cdot\left(f\left(y_{i}^{0}(s), y_{i}(s)\right)\left(1-\left|w_{i}(s)\right|\right)+\sum_{j=1}^{m} g_{j}\left(y_{i}^{0}(s), y_{i}(s)\right) w_{i}^{j}(s)\right)\right.\right. \\
\left.\left.\quad+p_{0 i}(s)\left(1-\left|w_{i}(s)\right|\right)+\pi_{i}\left|w_{i}(s)\right|\right]\left(1+d_{i}(s)\right)\right\} d s
\end{gathered}
$$

holds true for all measurable selectors $w$ and $d$ of $\left(1-\epsilon_{i}\right)\left(\mathcal{C} \cap \mathbb{B}_{m}\right)$ and $[-0.5,0.5]$ respectively, and, furthermore, one has the transversality relation

$$
\begin{aligned}
& \quad\left(p_{0 i}(0), p_{i}(0),-p_{0 i}(\bar{S}),-p_{i}(\bar{S}),-\pi_{i}\right) \\
& \in \partial\left(\phi\left(y_{i}^{0}(0), y_{i}(0), y_{i}^{0}(\bar{S}), y_{i}(\bar{S}), \nu_{i}(\bar{S})\right)+\rho_{i}\left|\left(y^{0}, y\right)(0)-\left(y_{i}^{0}, y_{i}\right)(0)\right|\right) \\
& \quad \subseteq \partial \phi\left(y_{i}^{0}(0), y_{i}(0), y_{i}^{0}(\bar{S}), y_{i}(\bar{S}), \nu_{i}(\bar{S})\right)+\rho_{i} \mathbb{B}_{1+n} \times\left\{0_{1+n}\right\} \times\{0\}
\end{aligned}
$$

Notice that we have set the cost multiplier equal to 1 , as is permitted, since $\left(P_{i}\right)$ has free right endpoint. It can be deduced from (27) and (29) that $\left\{\left(p_{0 i}, p_{i}\right)\right\}$ is an equi-bounded sequence of functions in $W^{1,1}$ with equi-integrable derivatives and $\left\{\pi_{i}\right\}$ is bounded. It follows that there exist $\left(p_{0}, p\right) \in W^{1,1}$ and $\pi \in \mathbb{R}$ such that, along some sequence (we do not relabel)

$$
\left\|\left(p_{0 i}, p_{i}\right)-\left(p_{0}, p\right)\right\|_{L^{\infty}(0, \bar{S})} \rightarrow 0, \quad \pi_{i} \rightarrow \pi \quad \text { as } i \rightarrow \infty .
$$

Notice next from (25) that

$$
\left|\left(y_{i}^{0}(0), y_{i}(0), y_{i}^{0}(\bar{S}), y_{i}(\bar{S}), \nu_{i}(\bar{S})\right)-\left(\bar{y}^{0}(0), \bar{y}(0), \bar{y}^{0}(\bar{S}), \bar{y}(\bar{S}), \bar{\nu}(\bar{S})\right)\right| \rightarrow 0 \quad \text { as } i \rightarrow \infty .
$$

Let $\left(\varphi_{i}^{0}, \varphi_{i}\right) \in W^{1,1}\left([0, \bar{S}] ; \mathbb{R} \times \mathbb{R}^{m}\right)$ be defined by

$$
\begin{array}{ll}
\varphi_{i}^{0}(s):=\bar{\varphi}^{0}(0)+\int_{0}^{s}\left(1+d_{i}(r)\right)\left(1-\left|w_{i}\right|(r)\right) d r & \\
\varphi_{i}(s):=\bar{\varphi}(0)+\int_{0}^{s}\left(1+d_{i}(r)\right) w_{i}(r) d r & \forall s \in[0, \bar{S}] .
\end{array}
$$

By the rate independence of the system

$$
\begin{aligned}
& \frac{d y_{i}^{0}}{d s}(s)=\left(1+d_{i}(s)\right) \frac{d \varphi_{i}^{0}}{d s}(s), \\
& \frac{d y_{i}}{d s}(s)=\left(1+d_{i}(s)\right)\left(f\left(y_{i}^{0}(s), y_{i}(s)\right) \frac{d \varphi_{i}^{0}}{d s}(s)+\sum_{j=1}^{m} g_{j}\left(y_{i}^{0}(s), y_{i}(s)\right) \frac{d \varphi_{i}^{j}}{d s}(s)\right), \\
& \frac{d \nu_{i}}{d s}(s)=\left(1+d_{i}(s)\right)\left|\frac{d \varphi_{i}}{d s}(s)\right|,
\end{aligned}
$$


by considering the reparameterization $\sigma_{i}(s)=\int_{0}^{s}\left(1+d_{i}\left(s^{\prime}\right)\right) d s^{\prime}$,

$$
\left(\tilde{y}_{i}^{0}, \tilde{y}_{i}, \tilde{\nu}_{i}, \tilde{\varphi}_{i}^{0}, \tilde{\varphi}_{i}\right)(\sigma):=\left(y_{i}^{0}, y_{i}, \nu_{i}, \varphi_{i}^{0}, \varphi_{i}\right)\left(\sigma_{i}^{-1}(\sigma)\right), \quad 0 \leq \sigma \leq \tilde{S},
$$

where $\tilde{S}_{i}:=\sigma_{i}(\bar{S})$, we obtain -see Remark 2.2 - that $\left(\tilde{y}_{i}^{0}, \tilde{y}_{i}, \tilde{\nu}_{i}\right)$ satisfies the differential system

$$
\begin{aligned}
& \frac{d \tilde{y}_{i}^{0}}{d s}(s)=\frac{d \tilde{\varphi}_{i}^{0}}{d s}(s) \quad \text { a.e. } s \in\left[0, \tilde{S}_{i}\right] \\
& \frac{d \tilde{y}_{i}}{d s}(s)=f\left(\tilde{y}_{i}^{0}(s), \tilde{y}_{i}(s)\right) \frac{d \tilde{\varphi}_{i}^{0}}{d s}(s)+\sum_{j=1}^{m} g_{j}\left(\tilde{y}_{i}^{0}(s), \tilde{y}_{i}(s)\right) \frac{d \tilde{\varphi}_{i}^{j}}{d s}(s) \quad \text { a.e. } s \in\left[0, \tilde{S}_{i}\right], \\
& \frac{d \tilde{\nu}_{i}}{d s}(s)=\left|\frac{d \tilde{\varphi}_{i}}{d s}(s)\right| \quad \text { a.e. } s \in\left[0, \tilde{S}_{i}\right] .
\end{aligned}
$$

We observe that, for each $i$, we have

$$
\begin{aligned}
& \frac{d \tilde{\varphi}_{i}^{0}}{d s}(s)=1-\left|\frac{d \tilde{\varphi}_{i}}{d s}(s)\right|, \quad \frac{d \tilde{\varphi}_{i}}{d s}(s) \in\left(1-\epsilon_{i}\right)\left(\mathcal{C} \cap \mathbb{B}_{m}\right) \text { a.e. } s \in\left[0, \tilde{S}_{i}\right] \\
& \left(\tilde{y}_{i}^{0}(0), \tilde{y}_{i}(0), \tilde{y}_{i}^{0}\left(\tilde{S}_{i}\right), \tilde{y}_{i}\left(\tilde{S}_{i}\right), \tilde{\nu}_{i}\left(\tilde{S}_{i}\right)\right)=\left(y_{i}^{0}(0), y_{i}(0), y_{i}^{0}(\bar{S}), y_{i}(\bar{S}), \nu_{i}(\bar{S})\right) .
\end{aligned}
$$

Therefore, we deduce from (25) that, for $i$ sufficiently large,

$$
d_{\infty}\left(\left(\tilde{y}_{i}^{0}(0), \tilde{y}_{i}^{0}\left(\tilde{S}_{i}\right), \tilde{y}_{i}, \tilde{\nu}_{i}\right),\left(\bar{y}^{0}(0), \bar{y}^{0}(\bar{S}), \bar{y}, \bar{\nu}\right)\right)<\delta,
$$

where $\delta>0$ is the constant appearing in Definition 4.2, with reference to the isolated extended sense feasible process $\left(\bar{S}, \bar{y}^{0}, \bar{y}, \bar{\nu}, \bar{\varphi}^{0}, \bar{\varphi}\right)$. Relations (31) and (32) tell us that $\left(\tilde{S}_{i}, \tilde{y}_{i}^{0}, \tilde{y}_{i}, \tilde{\nu}_{i}, \tilde{\varphi}_{i}^{0}, \tilde{\varphi}_{i}\right)$ is an embedded strict sense process. But taking note of the conditions imposed on the process $\left(\bar{S}, \bar{y}^{0}, \bar{y}, \bar{\nu}, \bar{\varphi}^{0}, \bar{\varphi}\right)$ in the theorem statement (namely, the fact that it is an isolated feasible extended sense process), we deduce from (34) that $\left(\tilde{S}_{i}, \tilde{y}_{i}^{0}, \tilde{y}_{i}, \tilde{\nu}_{i}, \tilde{\varphi}_{i}^{0}, \tilde{\varphi}_{i}\right)$ cannot be a feasible embedded strict sense process. We conclude that it must violate the endpoint constraints. By (33), also $\left(\bar{S}, y_{i}^{0}, y_{i}, \nu_{i}, \varphi_{i}^{0}, \varphi_{i}\right)$ must violate these constraints. Therefore,

$$
\max \left\{d_{\mathcal{T}}\left(y_{i}^{0}(0), y_{i}(0), y_{i}^{0}(\bar{S}), y_{i}(\bar{S})\right),\left(\nu_{i}(\bar{S})-K\right) \vee 0\right\}>0 .
$$

Inequality (35) provides the important information that, if the maximum in

$$
\phi\left(y_{i}^{0}(0), y_{i}(0), y_{i}^{0}(\bar{S}), y_{i}(\bar{S}), \nu_{i}(\bar{S})\right)=\max \left\{d_{\mathcal{T}}\left(y_{i}^{0}(0), y_{i}(0), y_{i}^{0}(\bar{S}), y_{i}(\bar{S})\right),\left(\nu_{i}(\bar{S})-K\right) \vee 0\right\}
$$

is achieved at $d_{\mathcal{T}}\left(y_{i}^{0}(0), y_{i}(0), y_{i}^{0}(\bar{S}), y_{i}(\bar{S})\right)$ then $d_{\mathcal{T}}\left(y_{i}^{0}(0), y_{i}(0), y_{i}^{0}(\bar{S}), y_{i}(\bar{S})\right)>0$, and if the maximum is achieved at $\left(\nu_{i}(\bar{S})-K\right) \vee 0$, then $\nu_{i}(\bar{S})-K>0$. Note also that (see, e.g., [27, Lemma 4.8.3])

$$
\begin{aligned}
& \partial d_{\mathcal{T}}\left(y_{i}^{0}(0), y_{i}(0), y_{i}^{0}(\bar{S}), y_{i}(\bar{S})\right) \subseteq \partial \mathbb{B}_{1+n+1+n} \quad \text { if }\left(y_{i}^{0}(0), y_{i}(0), y_{i}^{0}(\bar{S}), y_{i}(\bar{S})\right) \notin \mathcal{T}, \\
& \left.\partial\left(\nu_{i}(\bar{S})-K\right) \vee 0\right)=1 \quad \text { if } \nu_{i}(\bar{S})>K .
\end{aligned}
$$

Making use of these facts, we can deduce from the 'max' rule of subdifferential calculus (see, e.g., [27, Thm. 5.5.2]) the following estimate: if $\xi \in \partial \phi(z)$ at $z=\left(y_{i}^{0}(0), y_{i}(0), y_{i}^{0}(\bar{S}), y_{i}(\bar{S}), \nu_{i}(\bar{S})\right)$ then

$$
\xi \in \alpha_{i}\left(\left(\partial d_{\mathcal{T}}\left(y_{i}^{0}(0), y_{i}(0), y_{i}^{0}(\bar{S}), y_{i}(\bar{S})\right) \cap \partial \mathbb{B}_{1+n+1+n}\right) \times\{0\}\right)+\left(1-\alpha_{i}\right)\left(\left\{0_{1+n+1+n}\right\} \times\{1\}\right)
$$


with $\alpha_{i} \in[0,1]$. It follows then from (29) that

$$
\begin{aligned}
& \left(p_{0 i}(0), p_{i}(0),-p_{0 i}(\bar{S}),-p_{i}(\bar{S})\right) \in \\
& \quad \alpha_{i}\left(\partial d_{\mathcal{T}}\left(y_{i}^{0}(0), y_{i}(0), y_{i}^{0}(\bar{S}), y_{i}(\bar{S})\right) \cap \partial \mathbb{B}_{1+n+1+n}\right)+\rho_{i} \mathbb{B}_{1+n} \times\left\{0_{1+n}\right\}
\end{aligned}
$$

and

$$
\pi_{i}=-\left(1-\alpha_{i}\right) .
$$

By extracting further subsequences, we can arrange that $\alpha_{i} \rightarrow \alpha$ as $i \rightarrow \infty$, for some $\alpha \in[0,1]$. Passing to the limit in (27) (in 'integral' form), in (28) and in (29), as $i \rightarrow \infty$, with the help of (25), (26) and (30), we arrive at

$$
\begin{gathered}
-\frac{d p_{0}}{d s}(s)=p(s) \cdot\left(\frac{\partial f}{\partial t}\left(\bar{y}^{0}(s), \bar{y}(s)\right) \frac{d \bar{\varphi}^{0}}{d s}(s)+\sum_{j=1}^{m} \frac{\partial g_{j}}{\partial t}\left(\bar{y}^{0}(s), \bar{y}(s)\right) \frac{d \bar{\varphi}^{j}}{d s}(s)\right), \\
-\frac{d p}{d s}(s)=p(s) \cdot\left(\frac{\partial f}{\partial x}\left(\bar{y}^{0}(s), \bar{y}(s)\right) \frac{d \bar{\varphi}^{0}}{d s}(s)+\sum_{j=1}^{m} \frac{\partial g_{j}}{\partial x}\left(\bar{y}^{0}(s), \bar{y}(s)\right) \frac{d \bar{\varphi}^{j}}{d s}(s)\right) \text { a.e. } s \in[0, \bar{S}], \\
\int_{0}^{\bar{S}} p(s) \cdot\left(f\left(\bar{y}^{0}(s), \bar{y}(s)\right)(1-|w(s)|)+\sum_{j=1}^{m} g_{j}\left(\bar{y}^{0}(s), \bar{y}(s)\right) w^{j}(s)\right)(1+d(s)) d s \\
\quad+\int_{0}^{\bar{S}}\left(p_{0}(s)(1-|w(s)|)+\pi|w|\right)(1+d(s)) d s \\
\leq \int_{0}^{\bar{S}} p(s) \cdot\left(f\left(\bar{y}^{0}(s), \bar{y}(s)\right) \frac{d \bar{\varphi}^{0}}{d s}(s)+\sum_{j=1}^{m} g_{j}\left(\bar{y}^{0}(s), \bar{y}(s)\right) \frac{d \bar{\varphi}^{j}}{d s}(s)\right) d s \\
\quad+\int_{0}^{\bar{S}}\left(p_{0}(s) \frac{d \bar{\varphi}^{0}}{d s}(s)+\pi\left|\frac{d \bar{\varphi}^{0}}{d s}(s)\right|\right) d s,
\end{gathered}
$$

for all selectors $w$ and $d$ of $\mathcal{C} \cap \mathbb{B}_{m}$ and $[-0.5,0.5]$ respectively,

$$
\begin{aligned}
& \left(p^{0}(0), p(0),-p^{0}(\bar{S}),-p(\bar{S}) \in \alpha\left(\partial d_{\mathcal{T}}\left(\bar{y}^{0}(0), \bar{y}(0), \bar{y}^{0}(\bar{S}), \bar{y}(\bar{S})\right) \cap \partial \mathbb{B}_{1+n+1+n}\right),\right. \\
& |\pi|=1-\alpha .
\end{aligned}
$$

We deduce from (39) with the help of a measurable selection theorem that, for a.e. $s \in[0, \bar{S}]$,

$$
\begin{aligned}
& p(s) \cdot\left(f\left(\bar{y}^{0}(s), \bar{y}(s)\right) \frac{d \bar{\varphi}^{0}}{d s}(s)+\sum_{j=1}^{m} g_{j}\left(\bar{y}^{0}(s), \bar{y}(s)\right) \frac{d \bar{\varphi}^{j}}{d s}(s)\right)+p_{0}(s) \frac{d \bar{\varphi}^{0}}{d s}(s)+\pi\left|\frac{d \bar{\varphi}^{0}}{d s}(s)\right|= \\
& \max _{\left(w^{0}, w\right) \in \mathbf{C}, d \in[0.5,1.5]}\left\{\left[p(s) \cdot\left(f\left(\bar{y}^{0}(s), \bar{y}(s)\right) w^{0}+\sum_{j=1}^{m} g_{j}\left(\bar{y}^{0}(s), \bar{y}(s)\right) w^{j}\right)+p_{0}(s) w^{0}+\pi|w|\right](1+d)\right\} .
\end{aligned}
$$

Since 1 is interior to $[0.5,1.5]$, this implies

$$
\begin{aligned}
& p(s) \cdot\left(f\left(\bar{y}^{0}(s), \bar{y}(s)\right) \frac{d \bar{\varphi}^{0}}{d s}(s)+\sum_{j=1}^{m} g_{j}\left(\bar{y}^{0}(s), \bar{y}(s)\right) \frac{d \bar{\varphi}^{j}}{d s}(s)\right)+p_{0}(s) \frac{d \bar{\varphi}^{0}}{d s}(s)+\pi\left|\frac{d \bar{\varphi}^{0}}{d s}(s)\right|= \\
& \max _{\left(w^{0}, w\right) \in \mathbf{C}}\left\{p(s) \cdot\left(f\left(\bar{y}^{0}(s), \bar{y}(s)\right) w^{0}+\sum_{j=1}^{m} g_{j}\left(\bar{y}^{0}(s), \bar{y}(s)\right) w^{j}\right)+p_{0}(s) w^{0}+\pi|w|\right\}=0 \text { a.ф43) }
\end{aligned}
$$


Furthermore, (41) and (42) imply that

$$
\left(p_{0}(0), p(0),-p_{0}(\bar{S}),-p(\bar{S})\right) \in N_{\mathcal{T}}\left(\bar{y}^{0}(0), \bar{y}(0), \bar{y}^{0}(\bar{S}), \bar{y}(\bar{S})\right)
$$

From (41) and (42) we deduce that $\left(p_{0}, p, \pi\right) \neq(0,0,0)$. Employing the same arguments as those in the proof of $\mathrm{Thm}$. 3.1 we deduce from the latter relation that

$$
\left(p_{0}, p\right) \neq 0 \text {. }
$$

Surveying the relations satisfied by the absolutely continuous function $\left(p_{0}, p\right)$ and $\pi \leq 0$, namely (38), (43), (44) and (45), we see that the proof is complete.

\subsection{Proof of Theorem 4.1.}

It is convenient to prove the two assertons in reverse order.

(ii): Assume, contrary to the assertions of the theorem, that the $L^{\infty}$ local minimizer $\left(\bar{S}, \bar{y}^{0}, \bar{y}, \bar{\nu}, \bar{\varphi}^{0}, \bar{\varphi}\right)$ for $\left(P_{e}\right)$ is a normal extended sense extremal while, at the same time, there is a local infimum gap at $\left(\bar{S}, \bar{y}^{0}, \bar{y}, \bar{\nu}, \bar{\varphi}^{0}, \bar{\varphi}\right)$. We deduce from the latter property that there exists a number $c>0$ such that

$$
J_{e}\left(\bar{S}, \bar{y}^{0}, \bar{y}, \bar{\nu}, \bar{\varphi}^{0}, \bar{\varphi}\right)<J_{e}\left(S, y^{0}, y, \nu, \varphi^{0}, \varphi\right)-c
$$

for every embedded strict sense feasible process $\left(S, y^{0}, y, \nu, \varphi^{0}, \varphi\right)$ verifying (12) for the same $\delta>0$ as in Def. 2.5. Since $\left(\bar{S}, \bar{y}^{0}, \bar{y}, \bar{\nu}, \bar{\varphi}^{0}, \bar{\varphi}\right)$ is a normal extended sense extremal, we know from Thm. 4.2 that it is not an isolated extended sense feasible process. This means that there exists a sequence of embedded strict sense feasible processes $\left\{\left(S_{i}, y_{i}^{0}, y_{i}, \nu_{i}, \varphi_{i}^{0}, \varphi_{i}\right)\right\}$ such that

$$
\left|y_{i}^{0}(0)-\bar{y}^{0}(0)\right|+\left|y_{i}^{0}\left(S_{i}\right)-\bar{y}^{0}(\bar{S})\right|+\left\|\left(y_{i}, \nu_{i}\right)-(\bar{y}, \bar{\nu})\right\|_{L^{\infty}(\mathbb{R})} \rightarrow 0, \text { as } i \rightarrow \infty,
$$

(where $(\bar{y}, \bar{\nu})$ and $\left(y_{i}, \nu_{i}\right)$ are extended continuously to $\mathbb{R}$ by requiring them to be constant outside the original domains $[0, \bar{S}]$ and $\left[0, S_{i}\right]$, respectively). This is simply shown to imply that $\lim _{i \rightarrow \infty} S_{i}=\bar{S}$. But

$$
J_{e}\left(S, y_{i}^{0}, y_{i}, \nu_{i}, \varphi_{i}^{0}, \varphi_{i}\right)=h\left(y_{i}^{0}(0), y_{i}(0), y_{i}^{0}\left(S_{i}\right), y_{i}\left(S_{i}\right), \nu_{i}\left(S_{i}\right)\right)
$$

and, since $h$ is continuous, we deduce from (47) and (48) that

$$
\begin{aligned}
& \lim _{i \rightarrow \infty} J_{e}\left(S, y_{i}^{0}, y_{i}, \nu_{i}, \varphi_{i}^{0}, \varphi_{i}\right)=\lim _{i \rightarrow \infty} h\left(y_{i}^{0}(0), y_{i}(0), y_{i}^{0}\left(S_{i}\right), y_{i}\left(S_{i}\right), \nu_{i}\left(S_{i}\right)\right) \\
& \quad=h\left(\bar{y}^{0}(0), \bar{y}(0), \bar{y}^{0}(\bar{S}), \bar{y}(\bar{S}), \bar{\nu}(\bar{S})\right)=J_{e}\left(\bar{S}, \bar{y}^{0}, \bar{y}, \bar{\nu}, \bar{\varphi}^{0}, \bar{\varphi}\right) .
\end{aligned}
$$

This is not possible, in view of (46). So there is no local infimum gap at $\left(\bar{S}, \bar{y}^{0}, \bar{y}, \bar{\nu}, \bar{\varphi}^{0}, \bar{\varphi}\right)$. (i): Suppose that there exists a minimizer for $\left(P_{e}\right)$ (write it $\left(\bar{S}, \bar{y}^{0}, \bar{y}, \bar{\nu}, \bar{\varphi}^{0}, \bar{\varphi}\right)$ ) which is a normal extended sense extremal. Then $\left(\bar{S}, \bar{y}^{0}, \bar{y}, \bar{\nu}, \bar{\varphi}^{0}, \bar{\varphi}\right)$ is certainly an extended sense $L^{\infty}$ local minimizer. So by part (ii), there is no local infimum gap at $\left(\bar{S}, \bar{y}^{0}, \bar{y}, \bar{\nu}, \bar{\varphi}^{0}, \bar{\varphi}\right)$. This means that, for some $\delta>0$,

$$
J_{e}(\bar{z})=\inf \left\{J_{e}(z) \mid z \in \mathcal{A}_{e}^{+} \text {s.t. } d_{\infty}(z, \bar{z}) \leq \delta\right\} \geq \inf \left\{J_{e}(z) \mid z \in \mathcal{A}_{e}^{+}\right\},
$$


in which $\bar{z}:=\left(\bar{S}, \bar{y}^{0}, \bar{y}, \bar{\nu}, \bar{\varphi}^{0}, \bar{\varphi}\right)$. Since $\bar{z}$ is a minimizer,

$$
J_{e}(\bar{z})=\inf \left\{J_{e}(z) \mid z \in \mathcal{A}_{e}\right\}
$$

Bearing in that $\inf \left\{J_{e}(z) \mid z \in \mathcal{A}_{e}\right\} \leq \inf \left\{J_{e}(z) \mid z \in \mathcal{A}_{e}^{+}\right\}$, we conclude that

$$
\inf \left\{J_{e}(z) \mid z \in \mathcal{A}_{e}\right\}=\inf \left\{J_{e}(z) \mid z \in \mathcal{A}_{e}^{+}\right\}
$$

i.e. there is no infimum gap.

\section{Verifiable conditions for No Infimum Gap}

The sufficient condition of Thm. 4.1 for the absence of an infimum gap has the disadvantage, as a practical test, that it is expressed in terms of some minimizer, detailed information about which might not be available. The 'normality' test is of interest, nonetheless, because in certain special cases it can be replaced by simpler, verifiable conditions, some examples of which we now provide. These involve two notions of controllability w.r.t. the target set.

Definition 5.1. Consider the control system

$$
(S)\left\{\begin{array}{l}
\frac{d x}{d t}(t)=f(t, x(t))+\sum_{j=1}^{m} g_{j}(t, x(t)) \frac{d u^{j}}{d t}(t) \quad \text { a.e. } t \in\left[t_{1}, t_{2}\right], \\
\frac{d u}{d t}(t) \in \mathcal{C} \text { a.e. } t \in\left[t_{1}, t_{2}\right],
\end{array}\right.
$$

(i) $(S)$ is said to be quick 1-controllable w.r.t. the target set $\mathcal{T}$ at a point $\left(t_{1}, x_{1}, t_{2}, x_{2}\right) \in \mathcal{T}$ if, for any covector $\zeta=\left(\zeta_{t_{1}}, \zeta_{x_{1}}, \zeta_{t_{2}}, \zeta_{x_{2}}\right) \in N_{\mathcal{T}}\left(t_{1}, x_{1}, t_{2}, x_{2}\right)$ such that $\zeta_{x_{2}} \neq 0$, we have

$$
\inf _{w \in \mathcal{C}} \zeta_{x_{2}} \cdot \sum_{j=1}^{m} g_{j}\left(t_{2}, x_{2}\right) w^{j}<0 .
$$

(ii) $(S)$ is said to be drift-controllable w.r.t. the target set $\mathcal{T}$ at a point $\left(t_{1}, x_{1}, t_{2}, x_{2}\right) \in \mathcal{T}$ if, for any covector $\zeta=\left(\zeta_{t_{1}}, \zeta_{x_{1}}, \zeta_{t_{2}}, \zeta_{x_{2}}\right) \in N_{\mathcal{T}}\left(t_{1}, x_{1}, t_{2}, x_{2}\right)$ such that $\zeta_{x_{2}} \neq 0$, we have

$$
\zeta_{x_{2}} \cdot f\left(t_{2}, x_{2}\right)<0
$$

Proposition 5.1. Consider the optimal control problem $(P)$ and its extended sense formulation $\left(P_{e}\right)$. Let the data satisfy hypothesis (H1). Assume that there exists an extended sense minimizer $\left(\bar{S}, \bar{y}^{0}, \bar{y}, \bar{\nu}, \bar{\varphi}^{0}, \bar{\varphi}\right)$ such that

(i) $\bar{y}^{0}(\bar{S})>\bar{y}^{0}(0)$ (that is, the t-time interval is non-degenerate),

(ii) $\bar{\nu}(\bar{S})<K$,

(iii) $(S)$ is quick 1-controllable w.r.t. $\mathcal{T}$ at $\left(\bar{y}^{0}(0), \bar{y}(0), \bar{y}^{0}(\bar{S}), \bar{y}(\bar{S})\right)$.

Then $\left(\bar{S}, \bar{y}^{0}, \bar{y}, \bar{\nu}, \bar{\varphi}^{0}, \bar{\varphi}\right)$ is a normal extremal and, in consequence of Theorem 4.1, there is no infimum gap. 
Remark: The role of 1-quick controllability as a no infimum gap sufficient condition was earlier identified in [2], in the case when $\mathcal{T}$ takes the form

$$
\mathcal{T}=\{0\} \times\left\{x_{0}\right\} \times\{T\} \times \hat{\mathcal{T}},
$$

for some closed set $\hat{\mathcal{T}} \subset \mathbb{R}^{n}$ and some $T>0$. Prop. 5.1 broadens the applicability of the earlier sufficient condition by no longer requiring $\mathcal{T}$ to have this special structure.

Proof of Proposition [5.1. The proof involves showing that the given extended sense minimizer $\left(\bar{S}, \bar{y}^{0}, \bar{y}, \bar{\nu}, \bar{\varphi}^{0}, \bar{\varphi}\right)$ is a normal extremal; it will follow immediately from Thm. 4.1 that there is no infimum gap. Suppose that $\left(\bar{S}, \bar{y}^{0}, \bar{y}, \bar{\nu}, \bar{\varphi}^{0}, \bar{\varphi}\right)$ is not a normal extremal. Since, by assumption, $\bar{\nu}(\bar{S})<K$ and $\bar{y}^{0}(\bar{S})-\bar{y}^{0}(0)>0$, we can deduce from Thm. 3.1, there exists a set of multipliers $\left(p_{0}, p, \pi, \lambda\right)$, with $\lambda=0, p \neq 0$ and $\pi=0$. From (15) and (16) in Thm. 3.1 it may be deduced that

$$
\left(p_{0}(0), p(0), p_{0}(\bar{S}), p(\bar{S})\right)=\left(\zeta_{t_{1}}, \zeta_{x_{1}},-\zeta_{t_{2}},-\zeta_{x_{2}}\right)
$$

for some $\left(\zeta_{t_{1}}, \zeta_{x_{1}},-\zeta_{t_{2}},-\zeta_{x_{2}}\right) \in N_{\mathcal{T}}\left(\bar{y}^{0}(0), \bar{y}(0), \bar{y}^{0}(\bar{S}), \bar{y}(\bar{S})\right)$ with $\zeta_{x_{2}} \neq 0$.

From the almost everywhere condition (15) and the continuity of $\left(p_{0}, p\right)$ we deduce that

$$
\max _{\left(w^{0}, w\right) \in \mathbf{C}}\left\{-\zeta_{x_{2}} \cdot\left(f\left(\bar{y}^{0}(\bar{S}), \bar{y}(\bar{S})\right) w^{0}+\sum_{j=1}^{m} g_{j}\left(\bar{y}^{0}(\bar{S}), \bar{y}(\bar{S})\right) w^{j}\right)-\zeta_{t_{2}} w^{0}\right\}=0
$$

and, choosing $w_{0}=0$, we arrive at

$$
\min _{w \in \mathcal{C} \cap \partial \mathbb{B}_{m}} \zeta_{x_{2}} \cdot \sum_{j=1}^{m} g_{j}\left(\bar{y}^{0}(\bar{S}), \bar{y}(\bar{S})\right) w^{j} \geq 0 .
$$

This trivially violates the quick 1 -controllability hypothesis. So $\left(\bar{S}, \bar{y}^{0}, \bar{y}, \bar{\nu}, \bar{\varphi}^{0}, \bar{\varphi}\right)$ is a normal extremal.

If we assume $\mathcal{T}$ has an epigraph structure, as made precise below, then the assertions of Prop. 5.1 remain valid, either when it is no longer assumed that the $t$-time interval is non-degenerate (condition (i)), or when we replace (i)-(iii) by 'slow controllability'.

Proposition 5.2. Consider the optimal control problem $(P)$ and its extended sense formulation $\left(P_{e}\right)$. Let the data satisfy hypothesis (H1). Assume that there exists an extended sense minimizer $\left(\bar{S}, \bar{y}^{0}, \bar{y}, \bar{\nu}, \bar{\varphi}^{0}, \bar{\varphi}\right)$ such that

(i) $\mathcal{T}$ is an epigraph set on a neighborhood of $e:=\left(\bar{y}^{0}(0), \bar{y}(0), \bar{y}^{0}(\bar{S}), \bar{y}(\bar{S})\right)$, in the sense that there exist $\epsilon>0$ and a lower semicontinuous function $\psi: \mathbb{R} \times \mathbb{R}^{n} \times \mathbb{R}^{n} \rightarrow \mathbb{R}$ such that

$$
\mathcal{T} \cap\left(e+\epsilon \mathbb{B}_{1+2 n}\right)=\left\{\left(t_{1}, x_{1}, t_{2}, x_{2}\right) \mid t_{2} \geq \psi\left(t_{1}, x_{1}, x_{2}\right)\right\} \cap\left(e+\epsilon \mathbb{B}_{1+2 n}\right)
$$

(ii) either $(S)$ is drift-controllable w.r.t. $\mathcal{T}$ at $\left(\bar{y}^{0}(0), \bar{y}(0), \bar{y}^{0}(\bar{S}), \bar{y}(\bar{S})\right)$ or $\bar{\nu}(\bar{S})<K$ and system $(S)$ is quick 1-controllable w.r.t. $\mathcal{T}$ at $\left(\bar{y}^{0}(0), \bar{y}(0), \bar{y}^{0}(\bar{S}), \bar{y}(\bar{S})\right)$.

Then $\left(\bar{S}, \bar{y}^{0}, \bar{y}, \bar{\nu}, \bar{\varphi}^{0}, \bar{\varphi}\right)$ is a normal extended sense extremal and, in consequence of Thm. 4.1. there is no infimum gap. 
Proof. Once again, the proof involves showing that the given extended sense minimizer $\left(\bar{S}, \bar{y}^{0}\right.$, $\left.\bar{y}, \bar{\nu}, \bar{\varphi}^{0}, \bar{\varphi}\right)$ is a normal extremal; the fact that there is no infimum gap will then follow from Thm. 4.1. Suppose in contradiction that $\left(\bar{S}, \bar{y}^{0}, \bar{y}, \bar{\nu}, \bar{\varphi}^{0}, \bar{\varphi}\right)$ is not a normal extremal. To start with, notice that, since, by assumption, $\mathcal{T}$ is (locally) an epigraph set, we can deduce from Thm. 3.1 that there exists a set of multipliers $\left(p_{0}, p, \pi, \lambda\right)$, with $\lambda=0$ and $p \neq 0$. Indeed, if on the contrary $(p, \lambda)=(0,0)$, then (13) in Thm. 3.1 implies that $p_{0} \neq 0$. By the first equation in (14) we derive that $p_{0} \equiv \bar{p}_{0}$ is constant, from (15) it may be deduced that

$$
\bar{p}_{0} \leq 0
$$

while (16) implies that $\left(p_{0}(0), p(0), p_{0}(\bar{S}), p(\bar{S})\right)=\left(\zeta_{t_{1}}, \zeta_{x_{1}},-\zeta_{t_{2}},-\zeta_{x_{2}}\right)$ for some $\left(\zeta_{t_{1}}, \zeta_{x_{1}},-\zeta_{t_{2}},-\zeta_{x_{2}}\right) \in N_{\mathcal{T}}\left(\bar{y}^{0}(0), \bar{y}(0), \bar{y}^{0}(\bar{S}), \bar{y}(\bar{S})\right)$. Now, since $\mathcal{T}$ is (locally) an epigraph set, we know that $\zeta_{t_{2}} \leq 0$. Hence $\bar{p}_{0} \geq 0$, which together with (53) implies that $\bar{p}_{0}=0$, in contrast with the hypothesis $p_{0} \neq 0$. Thus there exists a set of multipliers $\left(p_{0}, p, \pi, \lambda\right)$, with $\lambda=0$ and $p \neq 0$.

At this point, from (15) and (16) in Thm. 3.1 it may be deduced that

$$
\left(p_{0}(0), p(0), p_{0}(\bar{S}), p(\bar{S})\right)=\left(\zeta_{t_{1}}, \zeta_{x_{1}},-\zeta_{t_{2}},-\zeta_{x_{2}}\right)
$$

for some $\left(\zeta_{t_{1}}, \zeta_{x_{1}},-\zeta_{t_{2}},-\zeta_{x_{2}}\right) \in N_{\mathcal{T}}\left(\bar{y}^{0}(0), \bar{y}(0), \bar{y}^{0}(\bar{S}), \bar{y}(\bar{S})\right)$ with $\zeta_{x_{2}} \neq 0$.

From (15) and the continuity of $\left(p_{0}, p\right)$ we deduce that

$$
\max _{\left(w^{0}, w\right) \in \mathbf{C}}\left\{-\zeta_{x_{2}} \cdot\left(f\left(\bar{y}^{0}(\bar{S}), \bar{y}(\bar{S})\right) w^{0}+\sum_{j=1}^{m} g_{j}\left(\bar{y}^{0}(\bar{S}), \bar{y}(\bar{S})\right) w^{j}\right)-\zeta_{t_{2}} w^{0}+\pi|w|\right\}=0 .
$$

Now, if $(S)$ is drift-controllable w.r.t. $\mathcal{T}$ at $\left(\bar{y}^{0}(0), \bar{y}(0), \bar{y}^{0}(\bar{S}), \bar{y}(\bar{S})\right)$, choosing $\left(w^{0}, w\right)=(1,0)$, we arrive at $-\zeta_{x_{2}} \cdot f\left(\bar{y}^{0}(\bar{S}), \bar{y}(\bar{S})\right)-\zeta_{t_{2}} \leq 0$, where $\zeta_{t_{2}} \leq 0$. This yields the contradiction to (50):

$$
\zeta_{x_{2}} \cdot f\left(\bar{y}^{0}(\bar{S}), \bar{y}(\bar{S})\right) \geq 0 .
$$

In case $\bar{\nu}(\bar{S})<K$ and system (S) is quick 1-controllable w.r.t. $\mathcal{T}$ at $\left(\bar{y}^{0}(0), \bar{y}(0), \bar{y}^{0}(\bar{S}), \bar{y}(\bar{S})\right)$, $\pi=0$ by Thm. 3.1. So, choosing $w_{0}=0$ in (54), we obtain the following contradiction to (49):

$$
\min _{w \in \mathcal{C} \cap \partial \mathbb{B}_{m}} \zeta_{x_{2}} \cdot \sum_{j=1}^{m} g_{j}\left(\bar{y}^{0}(\bar{S}), \bar{y}(\bar{S})\right) w^{j} \geq 0 .
$$

Hence $\left(\bar{S}, \bar{y}^{0}, \bar{y}, \bar{\nu}, \bar{\varphi}^{0}, \bar{\varphi}\right)$ is a normal extremal in both cases.

The no infimum gap sufficient conditions derived up to this point arise from the properties of normal extremals provided by Thm. 4.2, But normality-type conditions fail to cover some situations where we can demonstrate the absence of an infimum gap by independent analysis. One such case is identified in the following proposition.

Proposition 5.3 (The case without drift). Consider the optimal control problem $(P)$ and its extended sense formulation $\left(P_{e}\right)$. Let the data satisfy hypothesis (H1). Assume that

$$
f \equiv 0 \quad \text { ('no drift') } .
$$

Then there is no infimum gap. 
This sufficient condition is an immediate consequence of the lemma below, which yields directly the following information about systems with no drift: given an arbitrary extended sense process $\left(S, y^{0}, y, \nu, \varphi^{0}, \varphi\right)$, we can find a strict sense process $\left(t_{1}, t_{2}, x, v, u\right)$ with the same endpoints, that is

$$
y^{0}(0)=t_{1}, y(0)=x\left(t_{1}\right), y^{0}(S)=t_{2}, y(S)=x\left(t_{2}\right) \text { and } \nu(S)=v\left(t_{2}\right) .
$$

For such systems, an extended process is feasible if and only if the corresponding strict sense process is feasible and the costs (for problems $(P)$ and $\left(P_{e}\right)$ respectively) are the same; an infimum gap cannot then arise.

Lemma 5.1. Let hypothesis (H1) be satisfied. Assume that

$$
f \equiv 0 \text {. }
$$

Then, given any extended sense process $\left(S, y^{0}, y, \nu, \varphi^{0}, \varphi\right)$ for (8), there exists a strictly increasing, onto, absolutely continuous map $\sigma:\left[t_{1}, t_{2}\right] \rightarrow[0, S]$ such that the trajectory-control pair

$$
(x, v, u)(t):=(y, \nu, \varphi) \circ \sigma(t) \quad \forall t \in\left[t_{1}, t_{2}\right]
$$

is a strict sense process for (4).

Proof. Define

$$
\hat{\varphi}^{0}(s):=t_{1}+\frac{t_{2}-t_{1}}{S} s \quad \forall s \in[0, S] .
$$

Consider the bi-Lipschitz change of parameters $r:[0, S] \rightarrow\left[0, S^{\prime}\right]$

$$
r(s):=\int_{0}^{s}\left(\frac{d \hat{\varphi}^{0}}{d s^{\prime}}\left(s^{\prime}\right)+\left|\frac{d \varphi}{d s^{\prime}}\left(s^{\prime}\right)\right|\right) d s^{\prime} \quad \forall s \in[0, S]
$$

where $S^{\prime}:=r(S)$. Write the inverse mapping $s:=r^{-1}$. Now define the Lipschitz continuous functions

$$
\left(\tilde{\varphi}^{0}, \tilde{\varphi}\right)(r):=\left(\hat{\varphi}^{0}, \varphi\right) \circ s(r) \quad \forall r \in\left[0, S^{\prime}\right] .
$$

We can show, by means of straightforward calculations, that

(i) $\left(\frac{d \tilde{\varphi}^{0}}{d r}(r), \frac{d \tilde{\varphi}^{0}}{d r}(r)\right) \in \mathbf{C}$ for a.e. $r \in\left[0, S^{\prime}\right]$;

(ii) $\tilde{\varphi}^{0}(0)=t_{1}, \tilde{\varphi}^{0}\left(S^{\prime}\right)=t_{2}$;

(iii) the path $\left(\tilde{y}^{0}, \tilde{y}, \tilde{\nu}\right)$ defined by

$$
\left(\tilde{y}^{0}, \tilde{y}, \tilde{\nu}\right)(r):=\left(\tilde{\varphi}^{0}(r), y \circ s(r), \nu \circ s(r)\right) \quad \forall r \in\left[0, S^{\prime}\right]
$$

coincides with the unique solution to (8) corresponding to $\left(\tilde{\varphi}^{0}, \tilde{\varphi}\right)$ and initial state $\left(y^{0}, y, \nu\right)(0)$.

(Note that, to establish (iii), we make use of our assumption that $f \equiv 0$ ). Since $\frac{d \tilde{\varphi}^{0}}{d r}(r)>0$ for a.e. $r \in\left[0, S^{\prime}\right]$, the extended sense process $\left(S, \tilde{y}^{0}, \tilde{y}, \tilde{\nu}, \tilde{\varphi}^{0}, \tilde{\varphi}\right)$ is an embedded strict sense process, i.e., $(x, v, u)$ defined by

$$
(x, v, u)(t):=(\tilde{y}, \tilde{\nu}, \tilde{\varphi}) \circ\left(\tilde{\varphi}^{0}\right)^{-1}(t) \quad \forall t \in\left[t_{1}, t_{2}\right]
$$

is a strict sense process for (4) (in which $f \equiv 0$ ). To complete the proof, we observe that (55) follows from (56) and (57), when we choose $\sigma:=s \circ\left(\tilde{\varphi}^{0}\right)^{-1}$. 


\section{Examples}

We provide, in this section, a number of examples to illustrate the preceding theory.

Example 6.1. This example tells us that an infimum gap can actually occur when the sufficient condition of Thm. 4.1 is violated.

$$
\left\{\begin{array}{l}
\text { Minimize }-x_{1}(1) \\
\text { subject to } \\
\frac{d x_{1}}{d t}(t)=\frac{d u}{d t}(t) \text { a.e. } t \in[0,1] \\
\frac{d x_{2}}{d t}(t)=x_{1}(t) \text { a.e. } t \in[0,1] \\
\frac{d v}{d t}(t)=\left|\frac{d u}{d t}(t)\right| \text { a.e. } t \in[0,1] \\
\frac{d u}{d t}(t) \geq 0 \text { a.e. } t \in[0,1] \\
v(0)=0, v(1) \leq 1, x_{1}(0)=x_{2}(0)=0 \text { and } x_{2}(1) \leq 0 .
\end{array}\right.
$$

In this special case of $(P), n=2, m=1, \mathcal{T}=\{0\} \times\left\{0_{2}\right\} \times\{1\} \times \hat{\mathcal{T}}$, in which $\hat{\mathcal{T}}=\mathbb{R} \times(-\infty, 0]$, $\mathcal{C}=[0,+\infty), K=1$ and

$$
f(x)=\left(\begin{array}{c}
0 \\
x_{1}
\end{array}\right), g_{1}=\left(\begin{array}{l}
1 \\
0
\end{array}\right) \text {. }
$$

The extended problem is

$$
\left\{\begin{array}{l}
\text { Minimize }-y_{1}(S) \\
\text { over } S>0,\left(y^{0}, y, \nu, \varphi^{0}, \varphi\right) \in W^{1,1}\left([0, S] ; \mathbb{R} \times \mathbb{R}^{2} \times \mathbb{R} \times \mathbb{R} \times \mathbb{R}\right) \quad \text { satisfying } \\
\frac{d y^{0}}{d s}(s)=\frac{d \varphi^{0}}{d s}(s) \text { a.e. } s \in[0, S] \\
\frac{d y^{1}}{d s}(s)=\frac{d \varphi}{d s}(s) \quad \text { a.e. } s \in[0, S] \\
\frac{d y^{2}}{d s}(s)=y^{1}(s) \frac{d \varphi^{0}}{d s}(s) \quad \text { a.e. } s \in[0, S] \\
\frac{d \nu}{d s}(s)=\left|\frac{d \varphi}{d s}(s)\right| \quad \text { a.e. } s \in[0, S], \\
\left(\frac{d \varphi}{d s}(s), \frac{d \varphi}{d s}(s)\right) \in\left\{\left(w_{0}, w\right) \in \mathbb{R}_{+} \times \mathbb{R}_{+}: w_{0}+w=1\right\} \quad \text { a.e. } s \in[0, S], \\
\nu(0)=0, \nu(S) \leq 1,\left(y^{0}(0), y^{1}(0), y^{2}(0)\right)=(0,0,0), \quad y^{0}(S)=1, \quad y^{2}(S) \leq 0
\end{array}\right.
$$

It is straightforward to show that $\left(\bar{S}, \bar{y}^{0}, \bar{y}, \bar{\nu}, \bar{\varphi}^{0}, \bar{\varphi}\right)$ given by $\bar{S}=2, \frac{d \bar{\varphi}^{0}}{d s} \equiv 1-\bar{w}, \frac{d \bar{\varphi}}{d s} \equiv \bar{w}$, in which $\bar{y}=\left(\bar{y}^{1}, \bar{y}^{2}\right), \bar{y}_{2} \equiv 0, \bar{\nu} \equiv \bar{y}_{1}$ and

$$
\bar{y}_{1}(s)=\left\{\begin{array}{ll}
0 & \text { for } 0 \leq s \leq 1 \\
s-1 & \text { for } 1<s \leq 2
\end{array}, \bar{y}^{0}(s)=\left\{\begin{array}{ll}
s & \text { for } 0 \leq s \leq 1 \\
1 & \text { for } 1<s \leq 2
\end{array}, \bar{w}(s)= \begin{cases}0 & \text { for } 0 \leq s \leq 1 \\
1 & \text { for } 1<s \leq 2\end{cases}\right.\right.
$$


is a minimizer for the extended problem. Furthermore the infimum costs for the original and extended problems are, respectively,

$$
\inf (P)=0 \text { and } \inf \left(P_{e}\right)=-1
$$

Since there is an infimum gap, all the the sufficient conditions of the previous section, for non-occurence of an infimum gap, must be violated. In connection with Prop. 5.1 we note that the vector $\zeta=(0,1)$ lies in $N_{\hat{\mathcal{T}}}(\bar{y}(\bar{S}))=\{0\} \times[0, \infty)$ and

$$
\zeta \cdot g_{1} w \geq 0
$$

for every $w \in \mathcal{C}$, in violation of the the quick 1-controllability condition. (Also, condition $\bar{\nu}(\bar{S})<K$ is violated). Concerning the Prop. [5.2, we see that

$$
\zeta \cdot f(\bar{y}(\bar{S}))=0
$$

in violation of the drift controllability condition. Consider the normality condition. We can establish by simple calculations that $\left(\bar{S}, \bar{y}^{0}, \bar{y}, \bar{\nu}, \bar{\varphi}^{0}, \bar{\varphi}\right)$ is an extremal, and a possible Lagrange multiplier set is $\left(p_{0}, p_{1}, p_{2}, \pi, \lambda\right)$, in which

$$
p_{0} \equiv 0, \quad p_{1} \equiv 0, \quad p_{2} \equiv c, \quad \pi=0, \lambda=0,
$$

for any constant $c>0$. Notice that $\lambda=0$, so $\left(\bar{S}, \bar{y}^{0}, \bar{y}, \bar{\nu}, \bar{\varphi}^{0}, \bar{\varphi}\right)$ is an abnormal extremal. Since there is a unique minimizer 9 for the extended problem, we have shown that all minimizers for the extended problem are abnormal. Thus, the sufficient condition of Thm. 4.1 is violated.

Example 6.2. This example aims to demonstrate that the 'no infimum gap' sufficient condition of Thm. 4.1, based on normality, is distinct from those of Props. 5.1] and 5.2, based on quick 1-controllability and on drift controllability respectively. Consider the problem

$$
\left\{\begin{array}{l}
\text { Minimize } h(x(1)) \\
\text { over }(x, v, u) \in W^{1,1}\left([0,1] ; \mathbb{R}^{3} \times \mathbb{R} \times \mathbb{R}^{2}\right) \quad \text { satisfying } \\
\frac{d x}{d t}(t)=f(x(t))+g_{1}(x(t)) \frac{d u_{1}}{d t}(t)+g_{2}(x(t)) \frac{d u_{2}}{d t}(t), \quad \text { a.e. } t \in[0,1], \\
\frac{d v}{d t}(t)=\left|\frac{d u}{d t}(t)\right|, \quad \text { a.e. } t \in[0,1], \\
\frac{d u}{d t}(t) \in \mathcal{C}:=\mathbb{R}^{2} \quad \text { a.e. } t \in[0,1], \\
v(0)=0, \quad v(1) \leq K, \quad x(0)=(1,0,0), \quad x(1) \in \hat{\mathcal{T}}
\end{array}\right.
$$

in which $n=3, m=2, \mathrm{~K}=2, h(x):=-x_{1}, \hat{\mathcal{T}}:=\left\{\left(x_{1}, x_{2}, x_{3}\right) \mid x_{1} \leq 0, x_{2} \leq 0, x_{3} \leq 0\right\}$

$$
g_{1}(x):=\left(\begin{array}{c}
1 \\
0 \\
x_{2}
\end{array}\right), \quad g_{2}(x):=\left(\begin{array}{l}
0 \\
1 \\
-x_{1}
\end{array}\right), \quad f(x):=\left(\begin{array}{c}
0 \\
x_{2} \\
0
\end{array}\right), \quad \forall x \in \mathbb{R}^{3} .
$$

\footnotetext{
${ }^{9}$ Of course, 'unique' here means 'up to translations of $\left(\varphi^{0}, \varphi\right)$ '.
} 
This is an example of problem $(P)$, in which the underlying control system is a modification of the nonholonomic integrator, to include a non-zero drift term.

The extended problem is

$$
\left\{\begin{array}{l}
\text { Minimize } h(y(S)) \\
\text { over } S>0,\left(y^{0}, y, \nu, \varphi^{0}, \varphi\right) \in W^{1,1}\left([0, S] ; \mathbb{R} \times \mathbb{R}^{3} \times \mathbb{R} \times \mathbb{R} \times \mathbb{R}^{2}\right) \quad \text { satisfying } \\
\frac{d y^{0}}{d s}(s)=\frac{d \varphi^{0}}{d s}(s), \quad \text { a.e. } s \in[0, S], \\
\frac{d y}{d s}(s)=f(y(s)) \frac{d \varphi^{0}}{d s}(s)+g_{1}(y(s)) \frac{d \varphi^{1}}{d s}(s)+g_{2}(y(s)) \frac{d \varphi^{2}}{d s}(s), \quad \text { a.e. } s \in[0, S], \\
\frac{d \nu}{d s}(s)=\left|\frac{d \varphi}{d s}(s)\right|, \quad \text { a.e. } s \in[0, S], \\
\left(\frac{d \varphi^{0}}{d s}(s), \frac{d \varphi}{d s}(s)\right) \in \mathbf{C}:=\left\{\left(w_{0}, w\right) \in \mathbb{R}_{+} \times \mathbb{R}^{2}: w_{0}+|w|=1\right\} \quad \text { a.e. } s \in[0, S], \\
\nu(0)=0, \quad \nu(S) \leq K, \quad y(0)=(1,0,0), \quad y^{0}(0)=0, \quad y^{0}(S)=1, \quad y(S) \in \hat{\mathcal{T}} .
\end{array}\right.
$$

It is straighforward to show that the feasible extended sense process $\left(\bar{S}, \bar{y}^{0}, \bar{y}, \bar{\nu}, \bar{\varphi}^{0}, \bar{\varphi}\right)$, where $\bar{S}=2$,

$$
\left(\frac{d \bar{\varphi}^{0}}{d s}, \frac{d \bar{\varphi}}{d s}\right)=\left(\frac{d \bar{\varphi}^{0}}{d s}, \frac{d \bar{\varphi}^{1}}{d s}, \frac{d \bar{\varphi}^{2}}{d s}\right)=(1,0,0) \chi_{[0,1]}+(0,-1,0) \chi_{[1,2]}
$$

and

$$
\left(\bar{y}^{0}, \bar{y}, \bar{\nu}\right)=\left(\bar{y}^{0}, \bar{y}^{1}, \bar{y}^{2}, \bar{y}^{3}, \bar{\nu}\right)=(s, 1,0,0,0) \chi_{[0,1]}+(1,2-s, 0,0, s-1) \chi_{[1,2]},
$$

is the unique minimizer. By Thm. 3.1 there exists a set of multipliers $\left(p, p_{0}, \pi, \lambda\right)$ with $(p, \lambda) \neq 0$, where $\lambda \geq 0, p_{0} \in \mathbb{R}$ and $\pi=0$, since $\frac{\partial h}{\partial v} \equiv 0$ and $\bar{\nu}(\bar{S})=1<2$. The costate trajectory $p$ satisfies the differential system

$$
\left\{\begin{array}{l}
\frac{d p_{1}}{d s}(s)=0 \\
\frac{d p_{2}}{d s}(s)=-p_{2}(s) \chi_{[0,1]}(s)+p_{3}(s) \chi_{[1,2]}(s), \quad \text { a.e. } s \in[0,2] \\
\frac{d p_{3}}{d s}(s)=0
\end{array}\right.
$$

We see that $p_{1} \equiv \bar{p}_{1}$ and $p_{3} \equiv \bar{p}_{3}$ are constants. The triple $\left(p, p_{0}, \lambda\right)$ verifies the transversality condition

$$
-\left(p_{1}, p_{2}, p_{3}\right)(2) \in \lambda(-1,0,0)+N_{\hat{\mathcal{T}}}(0,0,0),
$$

and, for a.e. $s \in[0,2]$, the relations

$$
\begin{aligned}
& p_{0} \chi_{[0,1]}(s)-\bar{p}_{1} \chi_{[1,2]}(s)= \\
& \max _{\left(w_{0}, w\right) \in \mathbf{C}}\left\{p_{0} w_{0}+\bar{p}_{1} w_{1}+p_{2}(s)\left(\bar{y}_{2}(s) w_{0}+w_{2}\right)+\bar{p}_{3}\left(\bar{y}_{2}(s) w_{1}-\bar{y}_{1}(s) w_{2}\right)\right\}=0 .
\end{aligned}
$$


From the first relation in (65) we deduce that $p_{0}=\bar{p}_{1}=0$. Then, since $\bar{y}_{2} \equiv 0$ and $\left(w_{0}, w_{1}, w_{2}\right)=(0,0, \pm 1) \in \mathbf{C}$, by the second relation in (65) we necessarily have

$$
p_{2}(s)=\bar{p}_{3} \bar{y}_{1}(s) \quad \forall s \in[0,2] .
$$

Notice that $N_{\hat{\mathcal{T}}}(0,0,0)=\{(\alpha, \beta, \gamma)\}$ for any $\alpha, \beta$ and $\gamma \geq 0$. Hence condition (64) implies

$$
-\left(p_{1}, p_{2}, p_{3}\right)(2)=(-\lambda+\alpha, \beta, \gamma)
$$

and we arrive to the equalities

$$
\lambda=\alpha, \quad \bar{p}_{3}=-\gamma, \quad p_{2}=(\gamma-\beta) e^{1-s} \chi_{[0,1]}+(\gamma(2-s)-\beta) \chi_{[1,2]},
$$

It follows simply from (66) that $\gamma=\beta=0$, so that $\bar{p}_{3}=0$ and $p_{2} \equiv 0$. This proves that $\left(\bar{S}, \bar{y}^{0}, \bar{y}, \bar{\nu}, \bar{\varphi}^{0}, \bar{\varphi}\right)$ is a normal extremal, since $(p, \lambda) \neq 0$ if and only if $\lambda=\alpha>0$. We have shown that the conditions of Thm. 4.1 are satisfied and, in consequence, there is no infimum gap.

It is a straightforward matter to check that the quick 1-controllability and drift controllability conditions in Props. 5.1 and 5.2, respectively, are both violated. We know from Prop. 5.1 and Prop. 5.2, that the sufficient condition of Thm. 4.1 covers all cases when Props. 5.1] and 5.2 exclude infimum gaps. This example goes further, by showing that, in some cases, the sufficient condition of Thm. 4.1 excludes an infimum gap, when the other two conditions fail to do so and therefore has broader potential application.

Example 6.3. The purpose of this example is to demonstrate that the 'no infimum gap' sufficient condition of Thm. 4.1, based on normality, is not necessary. Consider the optimal control problem

$$
\left\{\begin{array}{l}
\operatorname{Minimize} h(x(1)) \\
\text { over }(x, v, u) \in W^{1,1}\left([0,1] ; \mathbb{R}^{3} \times \mathbb{R} \times \mathbb{R}^{2}\right) \quad \text { satisfying } \\
\frac{d x}{d t}(t)=g_{1}(x(t)) \frac{d u_{1}}{d t}(t)+g_{2}(x(t)) \frac{d u_{2}}{d t}(t), \quad \text { a.e. } t \in[0,1], \\
\frac{d v}{d t}(t)=\left|\frac{d u}{d t}(t)\right|, \quad \text { a.e. } t \in[0,1], \\
\frac{d u}{d t}(t) \in \mathcal{C}:=\left\{\left(w_{1}, w_{2}\right) \mid w_{1} \in \mathbb{R}, w_{2} \geq 0\right\} \quad \text { a.e. } t \in[0,1], \quad x(1) \in \hat{\mathcal{T}} \\
v(0)=0, \quad v(1) \leq K, \quad x(0)=(1,0,0), \quad x
\end{array}\right.
$$

in which $n=3, m=2, K=2, h$ and $g_{1}, g_{2}$ and $\hat{\mathcal{T}}$ are as in the previous example. Note 
however that, now, the drift term $f \equiv 0$. The extended problem is

$$
\left\{\begin{array}{l}
\text { Minimize } h(y(S)) \\
\text { over } S>0,\left(y^{0}, y, \nu, \varphi^{0}, \varphi\right) \in W^{1,1}\left([0, S] ; \mathbb{R} \times \mathbb{R}^{3} \times \mathbb{R} \times \mathbb{R} \times \mathbb{R}^{2}\right) \quad \text { satisfying } \\
\frac{d y^{0}}{d s}(s)=\frac{d \varphi^{0}}{d s}(s), \quad \text { a.e. } s \in[0, S], \\
\frac{d y}{d s}(s)=g_{1}(y(s)) \frac{d \varphi^{1}}{d s}(s)+g_{2}(y(s)) \frac{d \varphi^{2}}{d s}(s), \quad \text { a.e. } s \in[0, S] \\
\frac{d \nu}{d s}(s)=\left|\frac{d \varphi}{d s}(s)\right|, \quad \text { a.e. } s \in[0, S], \\
\left(\frac{d \varphi^{0}}{d s}(s), \frac{d \varphi}{d s}(s)\right) \in \mathbf{C}:=\left\{\left(w_{0}, w\right) \in \mathbb{R}_{+} \times \mathcal{C}: w_{0}+|w|=1\right\} \quad \text { a.e. } s \in[0, S], \\
\nu(0)=0, \quad \nu(S) \leq 2, \quad y(0)=(1,0,0), \quad y^{0}(0)=0, \quad y^{0}(S)=1, \quad\left(y_{1}, y_{2}, y_{3}\right)(S) \in \hat{\mathcal{T}}
\end{array}\right.
$$

The minimizing extended sense process $\left(\bar{S}, \bar{y}^{0}, \bar{y}, \bar{\nu}, \bar{\varphi}^{0}, \bar{\varphi}\right)$ for the optimal control problem (67), studied in the previous example, given by (61), (62), is a minimizing extended sense process also for problem (68). The multiplier set $\left(\lambda, p, p_{0}, \pi\right)$, where $(\lambda, p) \neq 0, \lambda \geq 0, p_{0} \in \mathbb{R}$, is such that $\pi=0$, since $\frac{\partial h}{\partial v} \equiv 0$ and $\bar{\nu}(\bar{S})=1<2$. The costate trajectory $p$ satisfies

$$
\left\{\begin{array}{l}
\frac{d p_{1}}{d s}(s)=0 \\
\frac{d p_{2}}{d s}(s)=p_{3}(s) \chi_{[1,2]}(s), \quad \text { a.e. } s \in[0,2] \\
\frac{d p_{3}}{d s}(s)=0
\end{array}\right.
$$

We see that $p_{1} \equiv \bar{p}_{1}$ and $p_{3} \equiv \bar{p}_{3}$ are constants. We also know that, for a.e. $s \in[0,2]$,

$$
p_{0} \chi_{[0,1]}(s)-\bar{p}_{1} \chi_{[1,2]}(s)=\max _{\left(w_{0}, w\right) \in \mathbf{C}}\left\{p_{0} w_{0}+\bar{p}_{1} w_{1}+p_{2}(s) w_{2}+\bar{p}_{3}\left(\bar{y}_{2}(s) w_{1}-\bar{y}_{1}(s) w_{2}\right)\right\}=0 .
$$

From (70) we deduce that $p_{0}=\bar{p}_{1}=0$ and, since $\bar{y}_{2} \equiv 0, \bar{y}_{1}=\chi_{[0,1]}+(2-s) \chi_{[1,2]}$ and $w_{2} \geq 0$ for all $\left(w_{0}, w\right) \in \mathbf{C}$, also that

$$
p_{2}(s) \leq \bar{p}_{3} \quad \forall s \in[0,1], \quad p_{2}(s) \leq \bar{p}_{3}(2-s) \quad \forall s \in[1,2] .
$$

We deduce from the transversality condition, as in the previous example, that

$$
-\left(p_{1}, p_{2}, p_{3}\right)(2)=(-\lambda+\alpha, \beta, \gamma)
$$

which implies $\lambda=\alpha \bar{p}_{3}=-\gamma$ and

$$
p_{2}=(\gamma-\beta) \chi_{[0,1]}+(\gamma(2-s)-\beta) \chi_{[1,2]} .
$$

(71) implies $\beta \geq 2 \gamma$. Choosing $\alpha=0$, and any $\gamma>0, \beta>2 \gamma$, we arrive at a multiplier set $\left(p_{0}=0, p_{1} \equiv 0, p_{2}, p_{3} \equiv-\gamma, \pi=0, \lambda=0\right)$, in which $p_{2}$ is given by (72). We have shown that 
the unique minimizer is not a normal extremal, so the sufficient condition for no infimum gap of Thm. 4.1 is not applicable to this problem.

Recall that the optimal control problem of this example has no drift. For such problems, we know that there can be no infimum gap. (See Prop. 5.3). Consequently, the sufficient condition of Thm. 4.1 fails to eliminate the possible occurrence of an infimum gap, in some circumstances when we can establish, by other means, that there is no infimum gap.

\section{References}

\section{References}

[1] M.S. Aronna, F. Rampazzo, $L^{1}$ limit solutions for control systems. J. Differential Equations 258, no. 3, 954-979, 2015.

[2] M.S. Aronna, M. Motta, F. Rampazzo, Infimum gaps for limit solutions. Set-Valued Var. Anal. 23, no. 1, 3-22, 2015.

[3] A. Arutyunov, D. Karamzin and F. Pereira, Pontryagin's maximum principle for constrained impulsive control problems. Nonlinear Anal. 75, no. 3, 10451057, 2012.

[4] A. Arutyunov, D. Karamzin and F. Pereira, A nondegenerate maximum principle for the impulse control problem with state constraints. SIAM J. Control Optim. 43, no. 5, $18121843,2005$.

[5] D. Azimov, R. Bishop (2005) New trends in astrodynamics and applications: optimal trajectories for space guidance. Ann. New York Acad. Sci. 1065(1), 189-209.

[6] Bonnans, J. F. and Shapiro, A. Perturbation Analysis of Optimization Problems, Springer, New York, 2000

[7] A.Bressan, F. Rampazzo, On differential systems with vector-valued impulsive controls. Boll. Un. Mat. Ital. B (7) 2, no. 3, 641-656, 1988.

[8] A. Bressan, B. Piccoli, Introduction to the mathematical theory of control. AIMS Series on Applied Mathematics, 2. American Institute of Mathematical Sciences (AIMS), Springfield, MO, 2007.

[9] A.Bressan, F. Rampazzo, Moving constraints as stabilizing controls in classical mechanics. Arch. Ration. Mech. Anal. 196, no. 1, 97-141, 2010.

[10] Aldo Bressan, Hyper-impulsive motions and controllizable coordinates for Lagrangean systems, Atti Accad. Naz. Lincei, Memorie, Serie VIII, Vol. XIX, 197-246, 1990.

[11] Aldo Bressan, On some control problems concerning the ski or swing, Atti Accad. Naz. Lincei, Memorie, Serie IX, Vol. I, 147-196, 1991.

[12] A. Catllá, D. Schaeffer, T. Witelski, E. Monson, A. Lin, (2008) On spiking models for synaptic activity and impulsive differential equations, SIAM Rev. 50(3), 553-569 
[13] F. H. Clarke, Y. S. Ledyaev, R. J. Stern and P. R. Wolenski, Nonsmooth Analysis and Control Theory, Graduate Texts in Mathematics vol. 178, Springer-Verlag, New York, 1998.

[14] A.L. Dontchev, T. Zolezzi, Well-posed optimization Springer-Verlag, New York, 1993.

[15] P. Gajardo, H. Ramirez C., A. Rapaport, Minimal time sequential batch reactors with bounded and impulse controls for one or more species, SIAM J. Control Optim. 47 (6) 2827-2856, 2008.

[16] M. Guerra, A. Sarychev, Fréchet generalized trajectories and minimizers for variational problems of low coercivity, J. Dyn. Control Syst. 21, no. 3, 351-377, 2015.

[17] O. Hájek, Discontinuous differential equations I, J. Differential Equations 32, 149-170, 1979.

[18] D.Y. Karamzin; V.A. de Oliveira, F.L. Pereira, G.N. Silva, On the properness of an impulsive control extension of dynamic optimization problems, ESAIM Control Optim. Calc. Var. 21, no. 3, 857-875, 2015.

[19] M. Miller, E. Y. Rubinovich, Impulsive control in continuous and discrete-continuous systems. Kluwer Academic/Plenum Publishers, New York, 2003.

[20] M. Motta, F. Rampazzo, Space-time trajectories of nonlinear systems driven by ordinary and impulsive controls. Differential Integral Equations 8, no. 2, 269-288, 1995.

[21] M. Motta, F. Rampazzo, Dynamic programming for nonlinear systems driven by ordinary and impulsive controls. SIAM J. Control Optim. 34, no. 1, 199-225, 1996.

[22] M. Motta, F. Rampazzo, State-constrained control problems with neither coercivity nor $L^{1}$ bounds on the controls. Ann. Mat. Pura Appl. (4) 177, 117-142, 1999.

[23] M. Motta, C. Sartori, On asymptotic exit-time control problems lacking coercivity. ESAIM Control Optim. Calc. Var. 20, no. 4, 957-982, 2014.

[24] R. T. Rockafellar and R. J.-B. Wets, Variational Analysis, Grundlehren der Mathematischen Wissenschaften, 317, Springer-Verlag, New York, 1998.

[25] G. Silva, R. Vinter, Measure driven differential inclusions, J. Math. Anal. Appl., 202 no. 3, 727-746, 1996.

[26] G. Silva, R. Vinter, Necessary conditions for optimal impulsive control problems. SIAM J. Control Optim. 35, no. 6, 1829-1846, 1997.

[27] R. B. Vinter, Optimal Control. Birkhäuser, Boston, 2000.

[28] M. Palladino and R. B. Vinter, Minimizers That Are Not Also Relaxed Minimizers, SIAM J. Control and Optim. 52, 4, 2164-2179, 2014.

[29] M. Palladino and R. B. Vinter, When are Minimizing Controls also Minimizing Relaxed Controls?, Discrete and Continuous Dyn. Systems Series A, 35, 4573-4592, 2015. 
[30] H.J. Sussmann, On the Gap Between Deterministic and Stochastic Ordinary Differential Equations The Annals of Probability, Vol. 6, No. 1, pp. 19-41

[31] F. Rampazzo, On the Riemannian structure of a Lagrangian system and the problem of adding time-dependent constraints as controls. European J. Mech. A Solids 10, no. 4, 405-431, 1991.

[32] J. Warga, Normal Control Problems have no Minimizing Strictly Original Solutions, Bulletin of the Amer. Math. Soc., 77, 4, 625-628, 1971.

[33] J. Warga, Optimal Control of Differential and Functional Equations, Academic Press, New York, 1972.

[34] P. Wolenski, S. Žabić, A differential solution concept for impulsive systems, Dyn. Contin. Discrete Impuls. Syst. Ser. A Math. Anal., 13B, 199-210, 2006. 